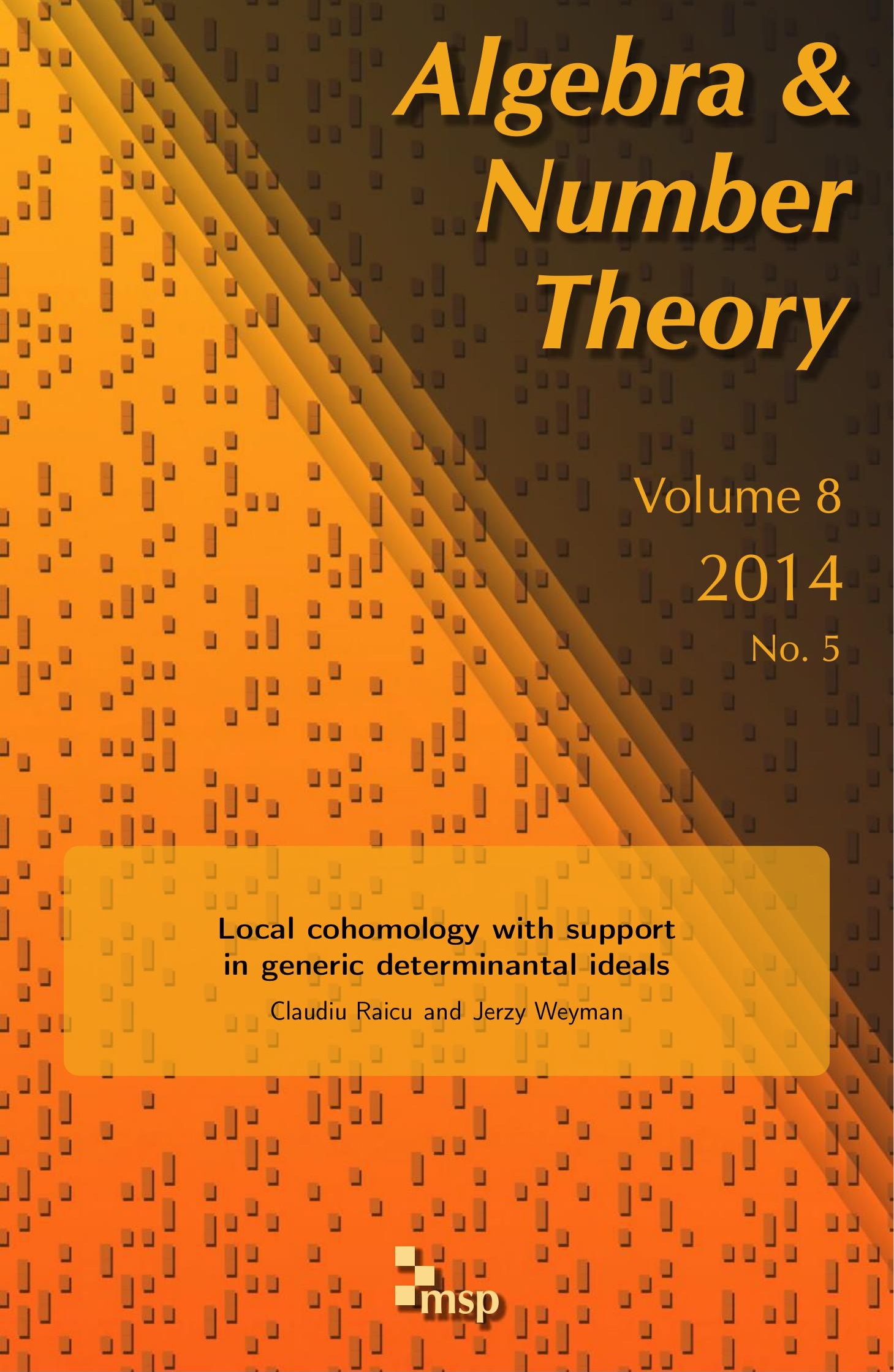




\title{
Local cohomology with support in generic determinantal ideals
}

\author{
Claudiu Raicu and Jerzy Weyman
}

To the memory of Andrei Zelevinsky

\begin{abstract}
For positive integers $m \geq n \geq p$, we compute the $\mathrm{GL}_{m} \times \mathrm{GL}_{n}$-equivariant description of the local cohomology modules of the polynomial $\operatorname{ring} S=\operatorname{Sym}\left(\mathbb{C}^{m} \otimes \mathbb{C}^{n}\right)$ with support in the ideal of $p \times p$ minors of the generic $m \times n$ matrix. Our techniques allow us to explicitly compute all the modules $\operatorname{Ext}_{S}^{\bullet}\left(S / I_{x}, S\right)$, for $\underline{x}$ a partition and $I_{x}$ the ideal generated by the irreducible subrepresentation of $S$ indexed by $\underline{x}$. In particular we determine the regularity of the ideals $I_{\underline{x}}$, and we deduce that the only ones admitting a linear free resolution are the powers of the ideal of maximal minors of the generic matrix, as well as the products between such powers and the maximal ideal of $S$.
\end{abstract}

\section{Introduction}

Given positive integers $m \geq n$ and a field $\mathbb{K}$ of characteristic zero, we consider the space $\mathbb{K}^{m \times n}$ of $m \times n$ matrices and the ring $S$ of polynomial functions on this space. For each $p=1, \ldots, n$ we define the ideal $I_{p} \subset S$ generated by the polynomial functions in $S$ that compute the $p \times p$ minors of the matrices in $\mathbb{K}^{m \times n}$. The goal of this paper is to describe for each $p$ the local cohomology modules $H_{I_{p}}^{\bullet}(S)$ of $S$ with support in the ideal $I_{p}$. The case $p=n$ was previously analyzed by the authors in joint work with Emily Witt [Raicu et al. 2014]. There is a natural action of the group $\mathrm{GL}_{m} \times \mathrm{GL}_{n}$ on $\mathbb{K}^{m \times n}$ and hence on $S$, and this action preserves each of the ideals $I_{p}$. This makes the $H_{I_{p}}^{\bullet}(S)$ into $\mathrm{GL}_{m} \times \mathrm{GL}_{n}$-representations, and our results describe the characters of these representations explicitly. Our methods also allow us to determine explicitly the characters of all the modules $\operatorname{Ext}_{S}^{\bullet}(S / I, S)$, where $I$ is an ideal of $S$ generated by an irreducible $\mathrm{GL}_{m} \times \mathrm{GL}_{n}$-subrepresentation of $S$, and in particular to determine the regularity of such ideals. It is an interesting problem to determine the minimal free resolutions of such ideals $I$, which unfortunately has

MSC2010: primary 13D45; secondary 14M12.

Keywords: local cohomology, determinantal ideals, regularity. 
only been answered in a small number of cases. We hope that our results will help shed some light on this problem in the future.

We will adopt a basis-independent notation throughout the paper, writing $F$ (resp. $G$ ) for a $\mathbb{K}$-vector space of dimension $m$ (resp. $n$ ), and thinking of $F^{*} \otimes G^{*}$ as the space $\mathbb{K}^{m \times n}$ of $m \times n$ matrices and of $S=\operatorname{Sym}(F \otimes G)$ as the ring of polynomial functions on this space. $S$ is graded by degree, with the space of linear forms $F \otimes G$ sitting in degree 1. The Cauchy formula [Weyman 2003, Corollary 2.3.3]

$$
S=\bigoplus_{\underline{x}=\left(x_{1} \geq \cdots \geq x_{n} \geq 0\right)} S_{\underline{x}} F \otimes S_{\underline{x}} G
$$

describes the decomposition of $S$ into a sum of irreducible $\operatorname{GL}(F) \times \operatorname{GL}(G)$ representations, indexed by partitions $\underline{x}$ with at most $n$ parts $\left(S_{\underline{x}}\right.$ denotes the Schur functor associated to $\underline{x}$ ). This decomposition respects the grading, the term corresponding to $\underline{x}$ being of degree $|\underline{x}|=x_{1}+\cdots+x_{n}$. We denote by $I_{\underline{x}}$ the ideal generated by $S_{\underline{x}} F \otimes S_{\underline{x}} G$. If we write $\left(1^{p}\right)$ for the partition $\underline{x}$ with $x_{1}=\cdots=x_{p}=1$ and $x_{i}=0$ for $i>p$, then $I_{\left(1^{p}\right)}$ is just another notation for the ideal $I_{p}$ of $p \times p$ minors. Our first result gives an explicit formula for the regularity of the ideals $I_{\underline{x}}$ :

Theorem 5.1 (regularity of equivariant ideals). For a partition $\underline{x}$ with at most $n$ parts, letting $x_{n+1}=-1$, we have the following formula for the regularity of the ideal $I_{\underline{x}}$ :

$$
\operatorname{reg}\left(I_{\underline{x}}\right)=\max _{\substack{p=1, \ldots, n \\ x_{p}>x_{p+1}}}\left(n \cdot x_{p}+(p-2) \cdot(n-p)\right) .
$$

In particular, the only ideals $I_{\underline{x}}$ which have a linear resolution are those for which $x_{1}=\cdots=x_{n}$ (i.e., powers $I_{n}^{x_{1}}$ of the ideal $I_{n}$ of maximal minors) or $x_{1}-1=$ $x_{2}=\cdots=x_{n}\left(\right.$ i.e., $\left.I_{n}^{x_{1}-1} \cdot I_{1}\right)$.

The minimal free resolutions of the powers of $I_{n}$ have been computed in [Akin et al. 1981, Theorem 5.4]. Together with the fact that $I \cdot \mathfrak{m}$ has a linear resolution whenever $I$ has a linear resolution and $\mathfrak{m}$ is the maximal homogeneous ideal, this implies that the ideals $I_{\underline{x}}$ have a linear resolution when $x_{2}=\cdots=x_{n}=x_{1}\left(\right.$ or $\left.x_{1}-1\right)$. The fact that no other $I_{\underline{x}}$ has a linear resolution is, to the best of our knowledge, new.

The theorem on the regularity of equivariant ideals is a consequence of the explicit description of the modules $\operatorname{Ext}_{S}^{\bullet}\left(S / I_{\underline{x}}, S\right)$ that we obtain in Theorem 4.3. This description is somewhat involved, so we avoid stating it for the moment. A key point is that the modules $\operatorname{Ext}_{S}^{\bullet}\left(S / I_{\underline{x}}, S\right)$ grow as we append new columns to the end of the partition $\underline{x}$. More precisely, we can identify a partition $\underline{x}$ with its pictorial realization as a Young diagram consisting of left-justified rows of boxes, 
with $x_{i}$ boxes in the $i$-th row; for example, $\underline{x}=(5,5,5,3)$ corresponds to

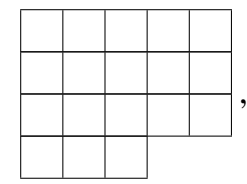

and adding two columns of size 2 and three columns of size 1 to the end of $\underline{x}$ yields $\underline{y}=(10,7,5,3)$.

Theorem 4.2 (the growth of Ext modules). Let $d \geq 0$ and consider partitions $\underline{x}, y$, where $\underline{x}$ consists of the first d columns of $\underline{y}$; i.e., $x_{i}=\min \left(y_{i}, d\right)$ for all $i=1, \ldots, n$. The natural quotient map $S / I_{y} \rightarrow S / I_{\underline{x}}$ induces injective maps

$$
\operatorname{Ext}_{S}^{i}\left(S / I_{\underline{x}}, S\right) \longleftrightarrow \operatorname{Ext}_{S}^{i}\left(S / I_{\underline{y}}, S\right)
$$

for all $i=0,1, \ldots, m \cdot n$.

We warn the reader that the naive generalization of the statement above fails: if $\underline{y}$ is a partition containing $\underline{x}$ (i.e., $y_{i} \geq x_{i}$ for all $i$ ), then it is not always the case that the induced maps $\operatorname{Ext}_{S}^{i}\left(S / I_{\underline{x}}, S\right) \rightarrow \operatorname{Ext}_{S}^{i}\left(S / I_{y}, S\right)$ are injective. In fact, a general partition $\underline{x}$ has the property that most modules $\operatorname{Ext}_{S}^{i}\left(S / I_{\underline{x}}, S\right)$ are nonzero, but it is always contained in some partition $\underline{y}$ with $y_{1}=\cdots=y_{n}$; for such a $\underline{y}$, all but $n$ of the modules $\operatorname{Ext}_{S}^{i}\left(S / I_{\underline{y}}, S\right)$ will vanish.

We next give the explicit description of $\operatorname{Ext}_{S}^{\bullet}\left(S / I_{\underline{x}}, S\right)$, which requires some notation. We write $\mathfrak{R}$ for the representation ring of the group $\operatorname{GL}(F) \times \operatorname{GL}(G)$. Given a $\mathbb{Z}$-graded $S$-module $M=\bigoplus_{i \in \mathbb{Z}} M_{i}$ admitting an action of $\operatorname{GL}(F) \times \operatorname{GL}(G)$ compatible with the natural one on $S$, we define its character $\chi_{M}(z)$ to be the element in the Laurent power series ring $\mathfrak{R}((z))$ given by

$$
\chi_{M}(z)=\sum_{i \in \mathbb{Z}}\left[M_{i}\right] \cdot z^{i},
$$

where $\left[M_{i}\right]$ denotes the class in $\Re$ of the $\operatorname{GL}(F) \times \operatorname{GL}(G)$-representation $M_{i}$. We will often work with doubly graded modules $M_{i}^{j}$, where the second grading (in $j$ ) is a cohomological one and $M_{\bullet}^{j} \neq 0$ for only finitely many values of $j$; for us they will be either Ext modules or local cohomology modules. We define the character of such an $M$ to be the element $\chi_{M}(z, w) \in \mathfrak{R}((z))\left[w^{ \pm 1}\right]$ given by

$$
\chi_{M}(z, w)=\sum_{i, j \in \mathbb{Z}}\left[M_{i}^{j}\right] \cdot z^{i} \cdot w^{j} .
$$

We will refer to an $r$-tuple $\lambda=\left(\lambda_{1}, \ldots, \lambda_{r}\right) \in \mathbb{Z}^{r}$ (for $r=m$ or $n$ ) as a weight. We say that $\lambda$ is dominant if $\lambda_{1} \geq \lambda_{2} \geq \cdots \geq \lambda_{r}$, and denote by $\mathbb{Z}_{\text {dom }}^{r}$ the set of dominant weights. Note that a partition is just a dominant weight with nonnegative entries. We will usually use the notation $\underline{x}, \underline{y}, \underline{z}$, etc. to refer to partitions indexing 
the subrepresentations of $S$, and $\lambda$, $\mu$, etc. to denote the weights describing the characters of other equivariant modules (Ext modules or local cohomology modules).

For $\lambda \in \mathbb{Z}_{\text {dom }}^{n}$ and $0 \leq s \leq n$, we define

$$
\lambda(s)=(\lambda_{1}, \ldots, \lambda_{s}, \underbrace{s-n, \ldots, s-n}_{m-n}, \lambda_{s+1}+(m-n), \ldots, \lambda_{n}+(m-n)) \in \mathbb{Z}^{m} .
$$

(Note that in [Raicu et al. 2014] this was called $\lambda(n-s)$.)

Theorem 4.3 (the characters of Ext Modules). With the above notation, the character of the doubly graded module $\operatorname{Ext}_{S}^{\bullet}\left(S / I_{\underline{x}}, S\right)$ is given by

$$
\begin{aligned}
& \chi_{\operatorname{Ext}_{S}^{\bullet}\left(S / I_{\underline{x}}, S\right)}(z, w) \\
& =\sum_{\substack{1 \leq p \leq n \\
0 \leq s \leq t_{1} \leq \cdots \leq t_{n-p} \leq p-1 \\
\lambda \in W^{\prime}(\underline{x}, p ; \underline{t}, s)}}\left[S_{\lambda(s)} F \otimes S_{\lambda} G\right] \cdot z^{|\lambda|} \cdot w^{m \cdot n+1-p^{2}-s \cdot(m-n)-2 \cdot\left(\sum_{j=1}^{n-p} t_{j}\right),}
\end{aligned}
$$

where $W^{\prime}(\underline{x}, p ; \underline{t}, s)$ is the set of dominant weights $\lambda \in \mathbb{Z}^{n}$ satisfying

$$
\left\{\begin{array}{l}
\lambda_{n} \geq p-x_{p}-m \\
\lambda_{t_{j}+j} \leq t_{j}-x_{n+1-j}-m \quad \text { for } j=1, \ldots, n-p, \\
\lambda_{s} \geq s-n \text { and } \lambda_{s+1} \leq s-m
\end{array}\right.
$$

Our proof of this theorem starts with the observation in [de Concini et al. 1980] that even though the algebraic set defined by $I_{\underline{x}}$ is somewhat simple (it is the set of matrices of rank smaller than the number of nonzero parts of $\underline{x}$ ), its scheme-theoretic structure is more complicated: it is generally nonreduced, and has embedded components supported on $I_{p}$ for each size $p$ of some column of $\underline{x}$. Our approach is then to filter $S / I_{\underline{x}}$ with subquotients $J_{\underline{z}, p}$ (defined in Section 2B) whose schemetheoretic support is the (reduced) space of matrices of rank at most $p$, which are therefore less singular and easier to resolve. In fact, each $J_{z, p}$ is the pushforward of a locally free sheaf on some product of flag varieties, which allows us to compute $\operatorname{Ext}_{S}^{\bullet}\left(J_{\underline{z}, p}, S\right)$ via duality theory. Solving the extension problem to deduce the formulas for $\operatorname{Ext}_{S}^{\bullet}\left(S / I_{\underline{x}}, S\right)$ turns out to be then trivial, due to the restrictions imposed by the equivariant structure of the modules.

We end this introduction with our main theorem on local cohomology modules, whose statement needs some more notation. For $0 \leq s \leq n$, we define (with the convention $\lambda_{0}=\infty, \lambda_{n+1}=-\infty$ )

$$
h_{s}(z)=\sum_{\substack{\lambda \in \mathbb{Z}_{\text {dom }}^{n} \\ \lambda_{s} \geq s-n \\ \lambda_{s+1} \leq s-m}}\left[S_{\lambda(s)} F \otimes S_{\lambda} G\right] \cdot z^{|\lambda|},
$$


so that $h_{n}(z)$ is just the character of $S$. The other $h_{s}(z)$ 's are characters of local cohomology modules with support in $I_{n}$ (in the case when $m>n$ ). More precisely, for $p=1, \ldots, n$ we write $H_{p}(z, w)$ for the character of the doubly graded module $H_{I_{p}}^{\bullet}(S)$. In [Raicu et al. 2014] we proved that for $m>n$

$$
H_{n}(z, w)=\sum_{s=0}^{n-1} h_{s}(z) \cdot w^{(n-s) \cdot(m-n)+1},
$$

and it is easy to see that the same formula holds for $m=n$ (in this case, the only nonzero local cohomology module is $H_{I_{n}}^{1}(S)=S_{\text {det }} / S$, where det denotes the determinant of the generic $n \times n$ matrix, and $S_{\text {det }}$ is the localization of $S$ at det).

We write $p(a, b ; c)$ for the number of partitions of $c$ contained in an $a \times b$ rectangle, and define the Gauss polynomial $\left(\begin{array}{c}a+b \\ b\end{array}\right)(w)$ to be the generating function for the sequence $p(a, b ; c)_{c \geq 0}$ :

$$
\left(\begin{array}{c}
a+b \\
a
\end{array}\right)(w)=\sum_{c \geq 0} p(a, b ; c) \cdot w^{c}=\sum_{b \geq t_{1} \geq t_{2} \geq \cdots \geq t_{a} \geq 0} w^{t_{1}+\cdots+t_{a}} .
$$

Gauss polynomials have previously appeared in [Akin and Weyman 2007] in connection to the closely related problem of understanding the minimal free resolutions of the ideals $I_{\left(p^{d}\right)}$.

Theorem 6.1 (local cohomology with support in generic determinantal ideals). With the above notation, we have, for each $p=1, \ldots, n$,

$$
H_{p}(z, w)=\sum_{s=0}^{p-1} h_{s}(z) \cdot w^{(n-p+1)^{2}+(n-s) \cdot(m-n)} \cdot\left(\begin{array}{c}
n-s-1 \\
p-s-1
\end{array}\right)\left(w^{2}\right) .
$$

The theorem implies that the maximal cohomological index for which $H_{I_{p}}^{\bullet}(S)$ is nonzero (the cohomological dimension of the ideal $I_{p}$ ) is obtained for $s=0$ and is equal to

$$
(n-p+1)^{2}+n \cdot(m-n)+(p-1) \cdot(n-p)=m \cdot n-p^{2}+1 .
$$

This was first observed in [Bruns and Schwänzl 1990]. Using the fact that once we invert one of the entries of a generic $m \times n$ matrix, $I_{p}$ becomes $I_{p-1}$ for a generic $(m-1) \times(n-1)$ matrix, it follows easily from the above that

$$
H_{I_{p}}^{j}(S) \neq 0 \quad \text { for } j=(m-s) \cdot(n-s)-(p-s)^{2}+1, s=0,1, \ldots, p-1 .
$$

For maximal minors $(p=n)$ this nonvanishing result is sharp, as explained in [Witt 2012]. Our next result, which is a direct consequence of Theorem 6.1, says that many more of the local cohomology modules $H_{I_{p}}^{j}(S)$ are nonzero when $p<n$, namely: 
Theorem (nonvanishing of local cohomology with determinantal support). If $p \leq$ $n \leq m$ then $H_{I_{p}}^{j}(S) \neq 0$ precisely when

$j=(n-p+1)^{2}+(n-s) \cdot(m-n)+2 \cdot k \quad$ for $0 \leq s \leq p-1,0 \leq k \leq(p-s-1) \cdot(n-p)$.

The nonvanishing statement (1-5) is obtained for $k=(p-s-1) \cdot(n-p)$.

This result contrasts with the positive characteristic situation, where the only nonvanishing local cohomology module appears in degree $j=(m-p+1)$. $(n-p+1)$ (see [Hochster and Eagon 1971, Corollary 4] or [Bruns and Vetter 1988, Corollary 5.18], where it is shown that $I_{p}$ is perfect, and [Peskine and Szpiro 1973, Proposition 4.1], where a local cohomology vanishing result for perfect ideals in positive characteristic is proved). For determinantal ideals over arbitrary rings one can't expect such explicit results as Theorem 6.1; for the latest advances in this general context, the reader should consult [Lyubeznik et al. 2013] and the references therein.

Our paper is organized as follows: In Section 2 we give some representationtheoretic preliminaries: in Section 2A we fix some notation for Schur functors, weights and partitions; in Section 2B we recall from [de Concini et al. 1980] some properties of the ideals $I_{\underline{x}}$ and introduce certain associated subquotients $J_{\underline{x}, p}$ that will play an important role in the sequel; in Section $2 \mathrm{C}$ we recall the definition of flag varieties and formulate some consequences of Bott's theorem in a form that will be useful to us; we also recall in Section 2D a method described in [Raicu et al. 2014] for computing extension groups for certain modules that arise as pushforwards of vector bundles with vanishing higher cohomology. In Section 3 we compute explicitly the characters of the modules $\operatorname{Ext}_{S}^{\bullet}\left(J_{\underline{x}, p}, S\right)$, and in Section 4 we use this calculation to deduce the main result about the characters of the modules $\operatorname{Ext}_{S}^{\bullet}\left(S / I_{\underline{x}}, S\right)$ for all partitions $\underline{x}$. In Section 5 we derive the formulas for the regularity of the ideals $I_{\underline{x}}$, while in Section 6 we describe the characters of the local cohomology modules with support in determinantal varieties.

\section{Preliminaries}

2A. Representation theory [Fulton and Harris 1991; Weyman 2003, Chapter 2]. Throughout the paper, $\mathbb{K}$ will denote a field of characteristic 0 . If $W$ is a $\mathbb{K}$-vector space of dimension $\operatorname{dim}(W)=N$, a choice of basis determines an isomorphism between $\mathrm{GL}(W)$ and $\mathrm{GL}_{N}(\mathbb{K})$. We will refer to $N$-tuples $\lambda=\left(\lambda_{1}, \ldots, \lambda_{N}\right) \in \mathbb{Z}^{N}$ as weights of the corresponding maximal torus of diagonal matrices. We say that $\lambda$ is a dominant weight if $\lambda_{1} \geq \lambda_{2} \geq \cdots \geq \lambda_{N}$. Irreducible (rational) representations of $\mathrm{GL}(W)$ are in one-to-one correspondence with dominant weights $\lambda$. We denote by $S_{\lambda} W$ the irreducible representation associated to $\lambda$, often referred to as the Schur functor. We write $\left(a^{N}\right)$ for the weight with all parts equal to $a$, and define 
the determinant of $W$ by $\operatorname{det}(W)=S_{\left(1^{N}\right)} W=\bigwedge^{N} W$. We have $S_{\lambda} W \otimes \operatorname{det}(W)=$ $S_{\lambda+\left(1^{N}\right)} W$ and $S_{\lambda} W^{*}=S_{\left(-\lambda_{N}, \ldots,-\lambda_{1}\right)} W$. We write $|\lambda|$ for the total size $\lambda_{1}+\cdots+\lambda_{N}$ of $\lambda$.

When $\underline{x}$ is a dominant weight with $x_{N} \geq 0$, we say that $\underline{x}$ is a partition of $r=|\underline{x}|$. Note that when we're dealing with partitions we often omit the trailing zeros, so $\underline{x}=(5,2,1)$ is the same as $\underline{x}=(5,2,1,0,0,0)$. If $\underline{y}$ is another partition, we write $\underline{x} \subset \underline{y}$ to indicate that $x_{i} \leq y_{i}$ for all $i$.

2B. The ideals $\boldsymbol{I}_{\underline{x}}$ and the subquotients $\boldsymbol{J}_{\underline{x}}, \boldsymbol{p}$. Recall the Cauchy formula (1-1) and the definition of the ideals $I_{\underline{x}} \subset S=\operatorname{Sym}(F \otimes G)$ as the ideals generated by subrepresentations $S_{\underline{x}} F \otimes S_{\underline{x}} G$ of $S$. It is shown in [de Concini et al. 1980] that

$$
I_{\underline{x}}=\bigoplus_{\underline{x} \subset \underline{y}} S_{\underline{y}} F \otimes S_{\underline{y}} G,
$$

and in particular $I_{y} \subset I_{\underline{x}}$ if and only if $\underline{x} \subset \underline{y}$. More generally, for arbitrary partitions $\underline{x}, \underline{y}$, we let $\underline{z}=\max (\underline{x}, \underline{y})$ be defined by $z_{i}=\max \left(x_{i}, y_{i}\right)$ for all $i$, and get

$$
I_{\underline{x}} \cap I_{\underline{y}}=I_{\underline{z}} .
$$

Even more generally, for any set $T$ of partitions we let

$$
I_{T}=\sum_{\underline{y} \in T} I_{\underline{y}}
$$

and have

$$
I_{\underline{x}} \cap I_{T}=\sum_{\underline{y} \in T} I_{\max (\underline{x}, \underline{y})} .
$$

For $p \in\{0,1, \ldots, n\}$ and $\underline{x}$ a partition, we write

$$
\operatorname{Succ}(\underline{x}, p)=\left\{\underline{y}: \underline{x} \subset \underline{y} \text {, and } y_{i}>x_{i} \text { for some } i>p\right\} .
$$

By the discussion above, $I_{\underline{y}} \subset I_{\underline{x}}$ for all $\underline{y} \in \operatorname{Succ}(\underline{x}, p)$. We define

$$
J_{\underline{x}, p}=I_{\underline{x}} / I_{\operatorname{Succ}(\underline{x}, p)}
$$

It follows from (2-1) that

$$
J_{\underline{x}, p}=\bigoplus_{\substack{\underline{x} \subset \underline{y} \\ y_{i}=x_{i} \text { for all } i>p}} S_{\underline{y}} F \otimes S_{\underline{y}} G .
$$

If $p=n$ then $J_{\underline{x}, p}=I_{\underline{x}}$, while if $p=0$ then $J_{\underline{x}, 0}=S_{\underline{x}} F \otimes S_{\underline{x}} G$, which as an $S$-module is annihilated by the maximal ideal of $S$. We have: 
Lemma 2.1. Fix an index $p \in\{0,1, \ldots, n-1\}$, and consider a partition $\underline{x}$ with $x_{1}=\cdots=x_{p+1}$. Let

$$
Z=\left\{\underline{z}: z_{1}=\cdots=z_{p+1}=x_{1}\right\} .
$$

We have

$$
I_{\operatorname{Succ}(\underline{x}, p)}=\left(\sum_{\underline{z} \in Z, \underline{x} \subseteq \underline{z}} I_{\underline{z}}\right)+I_{\max \left(\underline{x},\left(x_{1}+1\right)^{p+1}\right)} .
$$

Proof. " $\supset$ ": Consider $\underline{z} \in Z, \underline{x} \subsetneq \underline{z}$. We have $z_{i}>x_{i}$ for some $i$, and since $x_{i}=z_{i}$ for $i \leq p+1$, we conclude that $z_{i}>x_{i}$ for some $i>p+1$; thus, $\underline{z} \in \operatorname{Succ}(\underline{x}, p)$. Writing $\underline{y}=\max \left(\underline{x},\left(x_{1}+1\right)^{p+1}\right)$, we have that $y_{p+1}>x_{p+1}$ and $\underline{y} \supset \underline{x}$, so $\underline{y} \in \operatorname{Succ}(\underline{x}, p)$, proving that the right side of (2-9) is contained in the left.

" $\subset$ ": Consider a partition $\underline{y} \in \operatorname{Succ}(\underline{x}, p)$. If $y_{p+1}>x_{p+1}=x_{1}$ then $\underline{y}$ contains $\max \left(\underline{x},\left(x_{1}+1\right)^{p+1}\right)$, so $I_{y}$ is contained in the right side of (2-9). Otherwise $y_{p+1}=x_{p+1}$, so by possibly shrinking some of the first $p$ rows of $\underline{y}$ (which would enlarge $I_{y}$ ), we may assume that $y \in Z$. Clearly $y \supsetneq \underline{x}$, since $y_{i}>x_{i}$ for some $i>p+1$, so it follows again that $\bar{I}_{\underline{y}}$ is contained in the right side of (2-9).

The following result will be used in Section 4:

Lemma 2.2. Fix an index $p \in\{0,1, \ldots, n-1\}$, and consider a partition $\underline{x}$ with $x_{1}=$ $\cdots=x_{p+1}$. For a nonnegative integer $d \geq 0$, let $\underline{y}$ be the partition defined by $y_{i}=$ $x_{i}+d+1$ for $i=1, \ldots, p+1$ and $y_{i}=x_{i}$ for $i>p+1\left(\underline{y}=\max \left(\underline{x},\left(x_{1}+d+1\right)^{p+1}\right)\right)$. The quotient $I_{\underline{x}} / I_{\underline{y}}$ admits a filtration with successive quotients $J_{\underline{z}, p}$, where $\underline{z}$ runs over all partitions with

$$
\left\{\begin{array}{l}
x_{1} \leq z_{1}=\cdots=z_{p+1} \leq x_{1}+d \\
z_{i} \geq x_{i} \text { for } i>p+1
\end{array}\right.
$$

Proof. By induction, it suffices to prove the result when $d=0$. We consider $Z$ as in $(2-8)$ and define

$$
\mathscr{S}(Z)=\left\{I_{T}: T \subset Z\right\} .
$$

For $I \in \mathscr{I}(Z)$, we write

$$
Z(I)=\left\{\underline{z} \in Z: I_{\underline{z}} \subset I\right\} .
$$

Note that if $\underline{z}^{0} \in Z(I)$ then

$$
\text { if } \underline{z} \in Z \text { and } \underline{z}^{0} \subset \underline{z} \text { then } \underline{z} \in Z(I) .
$$

We let $I_{0}=I_{\left(\left(x_{1}+1\right)^{p+1}\right)}$ and prove by induction on $|Z(I)|$ that for $I \in \Phi(Z)$, the quotient $\left(I+I_{0}\right) / I_{0}$ has a filtration with successive quotients $J_{\underline{z}, p}$, where $\underline{z}$ varies 
over the set of elements of $Z(I)$. Once we do this, we can take $I=I_{\underline{x}}$ and observe that $I_{\underline{x}} \cap I_{0}=I_{y}$ (by (2-2)), which yields

$$
\left(I+I_{0}\right) / I_{0} \simeq I /\left(I \cap I_{0}\right)=I_{\underline{x}} / I_{\underline{y}},
$$

concluding the proof of the lemma.

For the induction, assume first that $|Z(I)|=1$, so that $I=I_{z}$ with $z_{1}=\cdots=$ $z_{n}=x_{1}$. We have $\left(I_{\underline{z}}+I_{0}\right) / I_{0}=J_{\underline{z}, p}$ so the base case for the induction follows.

Suppose now that $|Z(I)|>1$ and consider a maximal element $z^{0}$ in $Z(I)$, i.e., a partition $\underline{z}^{0}$ with the property that $I_{\underline{z}^{0}} \not \subset I_{\underline{z}}$ for any $\underline{z} \in Z(I) \backslash\left\{\underline{z}^{0}\right\}$. Define

$$
I^{\prime}=I_{Z(I) \backslash\left\{\underline{z}^{0}\right\}}
$$

and note that $\left|Z\left(I^{\prime}\right)\right|=|Z(I)|-1, I=I^{\prime}+I_{\underline{z}^{0}}$, and

$$
\left(I+I_{0}\right) /\left(I^{\prime}+I_{0}\right) \simeq J_{z^{0}, p},
$$

which is proved as follows. The equality $I=I^{\prime}+I_{\underline{z}^{0}}$ implies that the natural map

$$
I_{\underline{z}^{0}} \rightarrow\left(I+I_{0}\right) /\left(I^{\prime}+I_{0}\right)
$$

is surjective. Its kernel is

$$
\begin{aligned}
I_{\underline{z}^{0}} \cap\left(I^{\prime}+I_{0}\right) & \stackrel{(2-4)}{=}\left(\sum_{\underline{z} \in Z(I) \backslash\left\{\underline{z}^{0}\right\}} I_{\max \left(\underline{z}^{0}, \underline{z}\right)}\right)+I_{\max \left(\underline{z}^{0},\left(x_{1}+1\right)^{p+1}\right)} \\
& \stackrel{(2-10)}{=}\left(\sum_{\underline{z} \in Z, \underline{z}^{0} \subsetneq \underline{z}} I_{\underline{z}}\right)+I_{\max \left(\underline{z}^{0},\left(x_{1}+1\right)^{p+1}\right)} \stackrel{(2-9)}{=} I_{\operatorname{Succ}\left(\underline{z}^{0}, p\right),}
\end{aligned}
$$

from which (2-11) follows. Since by induction $\left(I^{\prime}+I_{0}\right) / I_{0}$ has a filtration with successive quotients $J_{\underline{z}, p}$ for $\underline{z} \in Z\left(I^{\prime}\right)$, we get the corresponding statement for $\left(I+I_{0}\right) / I_{0}$, finishing the induction step.

2C. Partial flag varieties and Bott's theorem [Weyman 2003, Chapter 4]. Consider a $\mathbb{K}$-vector space $V$ with $\operatorname{dim}(V)=d$ and positive integers $q \leq n \leq d$. We denote by $\operatorname{Flag}([q, n] ; V)$ the variety of partial flags

$$
V_{\bullet}: \quad V \rightarrow V_{n} \rightarrow V_{n-1} \rightarrow \cdots \rightarrow V_{q} \rightarrow 0,
$$

where $V_{p}$ is a $p$-dimensional quotient of $V$ for each $p=q, q+1, \ldots, n$. For $p$ in $[q, n]$ we write $2_{p}(V)$ for the tautological rank- $p$ quotient bundle on $\operatorname{Flag}([q, n] ; V)$ whose fiber over a point $V_{\bullet} \in \operatorname{Flag}([q, n] ; V)$ is $V_{p}$. For each $p$ there is a natural surjection of vector bundles

$$
V \otimes \mathcal{O}_{\mathrm{Flag}([q, n] ; V)} \rightarrow \mathscr{2}_{p}(V) .
$$


Note that for $q=n, \operatorname{Flag}([q, n] ; V)=\mathbb{G}(n, V)$ is the Grassmannian of $n$-dimensional quotients of $V$.

We consider the natural projection maps

$$
\pi^{(q)}: \operatorname{Flag}([q, n] ; V) \rightarrow \operatorname{Flag}([q+1, n] ; V),
$$

defined by forgetting $V_{q}$ from the flag $V_{\bullet}$. For $q \leq n-1$, this map identifies Flag $([q, n] ; V)$ with the projective bundle $\mathbb{P}_{\text {Flag }([q+1, n] ; V)}\left(2_{p+1}(V)\right)$, which comes with a tautological surjection

$$
2_{p+1}(V) \rightarrow 2_{p}(V) .
$$

For $q=n$ we make the convention Flag $([q+1, n]$; $V)=\operatorname{Spec}(\mathbb{K})$, so $\pi^{(n)}$ is just the structure map of $\mathbb{G}(n, V)$. With the usual notation $R^{\bullet} \pi_{*}^{(q)}$ for the derived push-forward, we obtain using [Weyman 2003, Corollary 4.1.9] the following:

Theorem 2.3. (a) Suppose that $q \leq n-1$, and consider a dominant weight $\mu \in \mathbb{Z}^{q}$. For $q<p \leq n$,

$$
R^{j} \pi_{*}^{(q)}\left(S_{\mu} \mathscr{2}_{p}(V)\right)= \begin{cases}S_{\mu} \mathscr{2}_{p}(V) & \text { if } j=0, \\ 0 & \text { otherwise. }\end{cases}
$$

If $\mu_{q-t}+t=-1$ for some $t=0, \ldots, q-1$, then

$$
R^{j} \pi_{*}^{(q)}\left(S_{\mu} \mathscr{2}_{q}(V)\right)=0 \text { for all } j .
$$

Otherwise (with the convention $\mu_{0}=\infty, \mu_{q+1}=-\infty$ ), consider the unique index $0 \leq t \leq q$ such that

$$
\mu_{q-t+1}+t+1 \leq 0 \leq \mu_{q-t}+t .
$$

Letting

$$
\tilde{\mu}=\left(\mu_{1}, \ldots, \mu_{q-t},-t, \mu_{q-t+1}+1, \ldots, \mu_{q}+1\right),
$$

we have

$$
R^{j} \pi_{*}^{(q)}\left(S_{\mu} \mathscr{Q}_{q}(V)\right)= \begin{cases}S_{\tilde{\mu}} \mathscr{2}_{q+1}(V) & \text { if } j=t, \\ 0 & \text { otherwise. }\end{cases}
$$

(b) Consider a dominant weight $\mu \in \mathbb{Z}^{n}$. If $n-d \leq \mu_{n-s}+s \leq-1$ for some $s=0, \ldots, n-1$, then

$$
R^{j} \pi_{*}^{(n)}\left(S_{\mu} \mathscr{Q}_{n}(V)\right)=0 \quad \text { for all } j .
$$

Otherwise (with the convention $\mu_{0}=\infty, \mu_{n+1}=-\infty$ ), consider the unique index $0 \leq s \leq n$ such that

$$
\mu_{n-s} \geq-s \quad \text { and } \quad \mu_{n-s+1} \leq-s-d+n .
$$




\section{Letting}

$$
\tilde{\mu}=(\mu_{1}, \ldots, \mu_{n-s}, \underbrace{-s, \ldots,-s}_{d-n}, \mu_{n-s+1}+(d-n), \ldots, \mu_{n}+(d-n)) \in \mathbb{Z}^{d},
$$

(compare to (1-2)), we have

$$
R^{j} \pi_{*}^{(n)}\left(S_{\mu} \mathscr{2}_{n}(V)\right)= \begin{cases}S_{\tilde{\mu}} V & \text { if } j=s \cdot(d-n), \\ 0 & \text { otherwise. }\end{cases}
$$

2D. Computing Ext modules via duality. In this section we recall [Raicu et al. 2014, Theorem 3.1] as a tool to compute $\operatorname{Ext}_{S}^{\bullet}(M, S)$ when $M$ comes as the pushforward of certain vector bundles with vanishing higher cohomology. More precisely, we have:

Theorem 2.4. Let $X$ be a projective variety, and let $W$ be a finite-dimensional $\mathbb{K}$-vector space. Suppose

$$
W \otimes \mathrm{O}_{X} \rightarrow \eta
$$

is a surjective map, where $\eta$ is locally free, and let $k=\operatorname{dim}(W)-\operatorname{rank}(\eta)$. Consider a locally free sheaf $\mathcal{V}$ on $X$, and define

$$
\mathcal{M}(\mathscr{V})=\mathscr{V} \otimes \operatorname{Sym}(\eta), \quad \mu^{*}(\mathscr{V})=\mathscr{V} \otimes \operatorname{det}(W) \otimes \operatorname{det}\left(\eta^{*}\right) \otimes \operatorname{Sym}\left(\eta^{*}\right) .
$$

Giving $\mathscr{V}$ internal degree $v$, and $\eta$ and $W$ degree 1 , we think of $\mathcal{M}(\mathscr{V})$ and $\mathcal{M}^{*}(\mathscr{V})$ as graded sheaves, with

$$
\mathcal{M}(\mathscr{V})_{i+v}=\mathscr{V} \otimes \operatorname{Sym}^{i}(\eta), \quad \mathcal{M}^{*}(\mathscr{V})_{i+v}=\mathscr{V} \otimes \operatorname{det}(W) \otimes \operatorname{det}\left(\eta^{*}\right) \otimes \operatorname{Sym}^{-i+k}\left(\eta^{*}\right) .
$$

Suppose that $H^{j}(X, M(\mathscr{V}))=0$ for $j>0$, and let

$$
M(\mathscr{V})=H^{0}(X, M(\mathscr{V})) .
$$

We have for each $j \geq 0$ a graded isomorphism

$$
\operatorname{Ext}_{S}^{j}(M(\mathscr{V}), S)=H^{k-j}\left(X, \mu^{*}(\mathscr{V})\right)^{*},
$$

where $(-)^{*}$ stands for the graded dual.

\section{Ext modules for the subquotients $J_{\underline{x}}, p$}

The goal of this section is to compute explicitly the character of $\operatorname{Ext}_{S}^{\bullet}\left(J_{\underline{x}}, p, S\right)$ for all $p$ and all partitions $\underline{x}$ with $x_{1}=\cdots=x_{p}$, where $J_{\underline{x}, p}$ is defined as in (2-6). We will achieve this by realizing $J_{\underline{x}, p}$ as the global sections of a vector bundle with vanishing higher cohomology on a certain product of flag varieties, and then using duality (Theorem 2.4) and Bott's theorem (Theorem 2.3). 
Consider as before vector spaces $F, G$, with $\operatorname{dim}(F)=m, \operatorname{dim}(G)=n, m \geq n$. For $q=1, \ldots, n$, we consider the projective varieties

$$
X^{(q)}=\operatorname{Flag}([q, n] ; F) \times \operatorname{Flag}([q, n] ; G), \quad X=X^{(\infty)}=\operatorname{Spec} \mathbb{K},
$$

and the locally free sheaves (see Section 2C)

$$
\eta^{(p)}=\mathscr{2}_{p}(F) \otimes \mathscr{2}_{p}(G), p=1, \ldots, n, \quad \eta=\eta^{(\infty)}=F \otimes G .
$$

Note that $\eta^{(p)}$ can be thought of as a sheaf on $X^{(q)}$ whenever $p \geq q$. We consider for $q \leq n-1($ resp. $q=n)$ the natural maps $\pi^{(q)}: X^{(q)} \rightarrow X^{(q+1)}\left(\right.$ resp. $\pi^{(n)}: X^{(n)} \rightarrow X$ ) induced from (2-13). We define

$$
S^{(q)}=\operatorname{Sym} \eta^{(q)}
$$

as relative versions of the polynomial ring $S=S^{(\infty)}=\operatorname{Sym}(F \otimes G)$. We will always work implicitly with quasicoherent sheaves on the affine bundles

$$
Y^{(q)}=\mathbb{A}_{X^{(q)}}\left(\eta^{(q)}\right)=\underline{\operatorname{Spec}}_{X^{(q)}}\left(S^{(q)}\right),
$$

which we identify with $S^{(q)}$-modules on $X^{(q)}$ as in [Hartshorne 1977, Exercise II.5.17]. The Cauchy formula (1-1) becomes in the relative setting

$$
S^{(q)}=\bigoplus_{\underline{x}=\left(x_{1} \geq \cdots \geq x_{q} \geq 0\right)} S_{\underline{x}} \mathscr{2}_{q}(F) \otimes S_{\underline{x}} \mathscr{2}_{q}(G),
$$

and we can define the ideals $I_{x}^{(q)} \subset S^{(q)}$ and subquotients $J_{x, p}^{(q)}$ for $0 \leq p \leq q$ analogously to (2-1) and (2-6). For $1 \leq p \leq q$, we write $I_{p}^{(q)}$ for $I_{\left(1^{p}\right)}^{(q)}$, the ideal of $p \times p$ minors in $S^{(q)}$. We define the line bundle

$$
\operatorname{det}^{(q)}=\operatorname{det}\left(\mathscr{Q}_{q}(F)\right) \otimes \operatorname{det}\left(\mathscr{2}_{q}(G)\right),
$$

and note that the ideal $I_{q}^{(q)}$ is generated by $\operatorname{det}^{(q)}$. It follows easily from (3-1) and Theorem 2.3 that

$$
R^{j} \pi_{*}^{(q)}\left(S^{(q)}\right)= \begin{cases}S^{(q+1)} / I_{q+1}^{(q+1)} & \text { if } j=0 \\ 0 & \text { otherwise }\end{cases}
$$

and, for $p>q$,

$$
R^{j} \pi_{*}^{(q)}\left(S^{(p)}\right)= \begin{cases}S^{(p)} & \text { if } j=0, \\ 0 & \text { otherwise. }\end{cases}
$$

Lemma 3.1. (a) For a partition $\underline{x}=\left(x_{1} \geq \cdots \geq x_{q}\right)$, there exist natural identifications

$$
\operatorname{det}^{(q)} \otimes I_{\underline{x}}^{(q)}=I_{\underline{x}+\left(1^{q}\right)}^{(q)},
$$

and

$$
\operatorname{det}^{(q)} \otimes J_{\underline{x}, p}^{(q)}=J_{\underline{x}+\left(1^{q}\right), p}^{(q)} \quad \text { for } 0 \leq p \leq q .
$$


(b) For a partition $\underline{x}=\left(x_{1} \geq \cdots \geq x_{q}\right)$, we have

$$
R^{j} \pi_{*}^{(q)} I_{\underline{x}}^{(q)}= \begin{cases}\left(I_{\underline{x}}^{(q+1)}+I_{q+1}^{(q+1)}\right) / I_{q+1}^{(q+1)} & \text { if } j=0, \\ 0 & \text { otherwise. }\end{cases}
$$

(c) For a partition $\underline{x}=\left(x_{1} \geq \cdots \geq x_{q}\right)$ and $0 \leq p \leq q$, we have

$$
R^{j} \pi_{*}^{(q)} J_{\underline{x}, p}^{(q)}= \begin{cases}J_{\underline{x}, p}^{(q+1)} & \text { if } j=0, \\ 0 & \text { otherwise. }\end{cases}
$$

Proof. (a) The multiplication map det ${ }^{(q)} \otimes S^{(q)} \rightarrow S^{(q)}$ is injective: if we think of $S^{(q)}$ as locally the ring of polynomial functions on $q \times q$ matrices, then $\operatorname{det}^{(q)}$ is the determinant of the generic $q \times q$ matrix. It follows that $\operatorname{det}^{(q)} \otimes I_{\underline{x}}^{(q)}=\operatorname{det}^{(q)} \cdot I_{\underline{x}}^{(q)}$ is in fact an ideal in $S^{(q)}$. Equation (3-4) then follows from the fact that multiplying by the determinant corresponds to adding a column of maximal size to the Young diagram (a special case of Pieri's rule). In fact, the same argument shows that for any set of partitions $Z$

$$
\operatorname{det}^{(q)} \otimes\left(\sum_{\underline{z} \in Z} I_{\underline{z}}^{(q)}\right)=\sum_{\underline{z} \in Z} I_{\underline{z}+\left(1^{q}\right)}^{(q)} .
$$

Given the definition of $J_{\underline{x}, p}^{(q)}$ as the analogue of (2-6), (3-5) follows by taking $Z=\operatorname{Succ}^{(q)}(\underline{x}, p)$ (the analogue of (2-5)) in the formula above, and using (3-4) and the exactness of tensoring with $\operatorname{det}^{(q)}$.

Part (b) follows from (3-3), while (c) follows from the fact that if $\underline{x}=\left(x_{1}, \ldots, x_{q}\right)$ and $0 \leq p \leq q$, then

$$
\operatorname{Succ}^{(q+1)}(\underline{x}, p)=\operatorname{Succ}^{(q)}(\underline{x}, p) \cup\left\{\underline{z}: \underline{z} \supset \underline{x}, z_{p+1} \geq 1\right\} .
$$

For each partition $\underline{x}=\left(x_{1}=\cdots=x_{p} \geq x_{p+1} \geq \cdots \geq x_{n} \geq x_{n+1}=0\right)$, we define the locally free sheaf $\mathcal{M}_{\underline{x}, p}$ on $X^{(p)}$ by

$$
\mu_{\underline{x}, p}=\left(\bigotimes_{q=p}^{n}\left(\operatorname{det}^{(q)}\right)^{\otimes\left(x_{q}-x_{q+1}\right)}\right) \otimes S^{(p)} .
$$

Lemma 3.2. With the notation above, we have

$$
H^{j}\left(X^{(p)}, \mu_{\underline{x}, p}\right)= \begin{cases}J_{\underline{x}, p} & \text { if } j=0, \\ 0 & \text { otherwise. }\end{cases}
$$

Proof. Note that $S^{(p)}=J_{\underline{0}, p}^{(p)}$, so using (3-4) we get

$$
\mu_{\underline{x}, p}=\left(\bigotimes_{q=p+1}^{n}\left(\operatorname{det}^{(q)}\right)^{\otimes\left(x_{q}-x_{q+1}\right)}\right) \otimes J_{\left(\left(x_{p}-x_{p+1}\right)^{p}\right), p}^{(p)} .
$$


It follows that

$$
\begin{aligned}
& R \pi_{*}^{(p)} \mu_{\underline{x}, p}=\pi_{*}^{(p)} \mu_{\underline{x}, p} \stackrel{(3-7)}{=}\left(\bigotimes_{q=p+1}^{n}\left(\operatorname{det}^{(q)}\right)^{\otimes\left(x_{q}-x_{q+1}\right)}\right) \otimes J_{\left(\left(x_{p}-x_{p+1}\right)^{p}\right), p}^{(p+1)} \\
& \stackrel{(3-4)}{=}\left(\bigotimes_{q=p+2}^{n}\left(\operatorname{det}^{(q)}\right)^{\otimes\left(x_{q}-x_{q+1}\right)}\right) \otimes J_{\left(\left(x_{p}-x_{p+2}\right)^{p}, x_{p+1}-x_{p+2}\right), p}^{(p+1)} .
\end{aligned}
$$

Applying $R \pi_{*}^{(p+1)}, R \pi_{*}^{(p+2)}, \ldots, R \pi_{*}^{(n)}$ iteratively, and using (3-7) and (3-4) as above, we obtain

$$
R \pi_{*} M_{\underline{x}, p}=\pi_{*} M_{\underline{x}, p}=J_{\left(x_{p}^{p}, x_{p+1}, \ldots, x_{n}\right), p} \stackrel{\left(x_{1}=\cdots=x_{p}\right)}{=} J_{\underline{x}, p},
$$

where $\pi=\pi^{(n)} \circ \cdots \circ \pi^{(p)}$ is the structure map $X^{(p)} \rightarrow$ Spec $\mathbb{K}$, concluding the proof of the lemma.

We are now ready to prove the main result of this section:

Theorem 3.3. The character of the doubly graded module $\operatorname{Ext}_{S}^{\bullet}\left(J_{\underline{x}}, p, S\right)$ is given by

$$
\begin{aligned}
\chi_{\operatorname{Ext}_{S}^{\bullet}\left(J_{\underline{x}, p}, S\right)}(z, w) & \sum_{\substack{0 \leq s \leq t_{1} \leq \ldots \leq t_{n-p} \leq p \\
\lambda \in W(\underline{x}, p ; \underline{t}, s)}}\left[S_{\lambda(s)} F \otimes S_{\lambda} G\right] \cdot z^{|\lambda|} \cdot w^{m \cdot n-p^{2}-s \cdot(m-n)-2 \cdot\left(\sum_{j=1}^{n-p} t_{j}\right)},
\end{aligned}
$$

where $W(\underline{x}, p ; \underline{t}, s)$ is the set of dominant weights $\lambda \in \mathbb{Z}^{n}$ with the properties

$$
\left\{\begin{array}{l}
\lambda_{n} \geq p-x_{p}-m, \\
\lambda_{t_{j}+j}=t_{j}-x_{n+1-j}-m \quad \text { for } j=1, \ldots, n-p, \\
\lambda_{s} \geq s-n \quad \text { and } \quad \lambda_{s+1} \leq s-m .
\end{array}\right.
$$

Remark 3.4. If we take $p=n$ and $x_{1}=\cdots=x_{n}=d$ in the above theorem, we recover [Raicu et al. 2014, Theorem 4.3]. The character of $J_{\underline{x}, n}=I_{\underline{x}}=I_{n}^{d}$ is

$$
\chi_{\operatorname{Ext}_{S}^{\bullet}\left(J_{\underline{x}, n}, S\right)}(z, w)=\sum_{\substack{0 \leq s \leq n \\ \lambda_{n} \geq n-d-m \\ \lambda_{s} \geq s-n \\ \lambda_{s+1} \leq s-m}}\left[S_{\lambda(s)} F \otimes S_{\lambda} G\right] \cdot z^{|\lambda|} \cdot w^{(n-s) \cdot(m-n)} .
$$

When $p=0$, since $J_{\underline{x}, 0}=S_{\underline{x}} F \otimes S_{\underline{x}} G$ is just a vector space the only nonvanishing Ext module is

$$
\operatorname{Ext}_{S}^{m n}\left(J_{\underline{x}, 0}, S\right)=\left(S_{\underline{x}} F \otimes S_{\underline{x}} G \otimes \operatorname{det}(F \otimes G)\right)^{*} .
$$


Proof of Theorem 3.3. We apply Theorem 2.4 with

$$
X=X^{(p)}, \quad \eta=\eta^{(p)}, \quad W=F \otimes G, \quad \mathcal{V}=\bigotimes_{q=p}^{n}\left(\operatorname{det}^{(q)}\right)^{\otimes\left(x_{q}-x_{q+1}\right)},
$$

so that $\mathcal{M}(\mathscr{V})=M_{\underline{x}, p}$ (see (3-8)). Lemma 3.2 ensures that the hypotheses of the duality theorem hold, and $M(\mathscr{V})=J_{\underline{x}, p}$. We have $\operatorname{rank}\left(\eta^{(p)}\right)=p^{2}, \operatorname{dim}(W)=m \cdot n$, so $k=m \cdot n-p^{2}$. We give $\mathcal{V}$ internal degree $v=|\underline{x}|$, and get

$\operatorname{Ext}_{S}^{j}\left(J_{\underline{x}, p}, S\right)_{r-|\underline{x}|}$

$$
\begin{aligned}
=H^{m \cdot n-p^{2}-j}\left(X^{(p)}, \bigotimes_{q=p}^{n}\left(\operatorname{det}^{(q)}\right)^{\otimes\left(x_{q}-x_{q+1}\right)} \otimes \operatorname{det}(F \otimes G)\right. \\
\left.\otimes \operatorname{det}\left(\eta^{*}\right) \otimes \operatorname{Sym}^{r+m \cdot n-p^{2}}\left(\eta^{*}\right)\right)^{*} .
\end{aligned}
$$

Formula (3-9) now follows from a direct application of Theorem 2.3, which we sketch below.

Using Cauchy's formula and that $\operatorname{det}\left(\eta^{*}\right)=\operatorname{det}\left(2_{p}(F)\right)^{-p} \otimes \operatorname{det}\left(\mathscr{2}_{p}(G)\right)^{-p}$, we get

$$
\operatorname{det}\left(\eta^{*}\right) \otimes \operatorname{Sym}^{r+m \cdot n-p^{2}}\left(\eta^{*}\right)=\bigoplus_{\substack{\mu \in \mathbb{Z}_{\text {dom }}^{p} \\|\mu|=r+m \cdot n \\ \mu_{1} \leq-p}} S_{\mu} \mathscr{2}_{p}(F) \otimes S_{\mu} \mathscr{2}_{p}(G) .
$$

For each $\mu$ in the formula above, we have to first compute

$$
R \pi_{*}\left(\bigotimes_{q=p}^{n}\left(\operatorname{det}^{(q)}\right)^{\otimes\left(x_{q}-x_{q+1}\right)} \otimes S_{\mu} \mathscr{2}_{p}(F) \otimes S_{\mu} \mathscr{2}_{p}(G)\right),
$$

where $\pi=\pi^{(n)} \circ \cdots \circ \pi^{(p)}: X^{(p)} \rightarrow$ Spec $\mathbb{K}$ is the structure map, then tensor with $\operatorname{det}(F \otimes G)$ and dualize, in order to get the corresponding contribution to (3-11). If (3-12) is nonzero, then there exist uniquely determined dominant weights $\mu^{(q)}, \delta^{(q)} \in \mathbb{Z}^{q}$ for $q=p, \ldots, n$, and nonnegative integers $t_{n-q}, q=p, \ldots, n-1$, and $s$, such that $\mu^{(p)}=\mu$, and if for $q=p, \ldots, n$ we write

$$
\mu^{(q)}=S_{\mu^{(q)}} \mathscr{2}_{q}(F) \otimes S_{\mu^{(q)}} \mathscr{2}_{q}(G), \quad \mathcal{N}^{(q)}=S_{\delta(q)} \mathscr{2}_{q}(F) \otimes S_{\delta(q)} \mathscr{2}_{q}(G),
$$

then

$$
\mathcal{N}^{(q)}=\mathcal{M}^{(q)} \otimes\left(\operatorname{det}^{(q)}\right)^{\otimes\left(x_{q}-x_{q+1}\right)} \quad \text { for } q=p, \ldots, n,
$$

$2 \cdot t_{n-q}$ is the unique integer $j$ for which $R^{j} \pi_{*}^{(q)}\left(\mathcal{N}^{(q)}\right) \neq 0$, and

$$
R^{2 \cdot t_{n-q}} \pi_{*}^{(q)}\left(\mathcal{N}^{(q)}\right)=\mathcal{M}^{(q+1)} \text { for } q=p, \ldots, n-1,
$$

and finally, $s \cdot(m-n)$ is the unique integer $j$ for which $R^{j} \pi_{*}^{(n)}\left(\mathcal{N}^{(n)}\right) \neq 0$. 
The dominant weight $\delta^{(q)}$ is easy to determine; namely, we get from (3-13) that

$$
\delta^{(q)}=\mu^{(q)}+\left(\left(x_{q}-x_{q+1}\right)^{q}\right) .
$$

Assuming we know $\delta^{(q)}$, (3-14) determines $t_{n-q}$ and $\mu^{(q)}$ according to (a) of Theorem 2.3: $t_{n-q}$ is the unique integer $t$ with the property

$$
\delta_{q-t+1}^{(q)}+t+1 \leq 0 \leq \delta_{q-t}^{(q)}+t
$$

and

$$
\mu^{(q+1)}=\left(\delta_{1}^{(q)}, \ldots, \delta_{q-t_{n-q}}^{(q)},-t_{n-q}, \delta_{q-t_{n-q}+1}^{(q)}+1, \ldots, \delta_{q}^{(q)}+1\right) .
$$

It follows from (3-17) and (3-15) that

$$
\delta_{q+1-t_{n-q}}^{(q+1)}=-t_{n-q}+x_{q+1}-x_{q+2} \geq-t_{n-q},
$$

so $t=t_{n-q}$ satisfies the right-hand inequality in (3-16) with $q$ replaced by $(q+1)$, which forces $t_{n-(q+1)} \leq t_{n-q}$. It follows easily that

$$
\delta_{q+1-t_{n-q}}^{(i)}=-t_{n-q}+x_{q+1}-x_{i+1} \quad \text { for } i=q+1, \ldots, n .
$$

We have seen so far how to calculate $\mu^{(q)}, \delta^{(q)}$ for $q=p, \ldots, n$, and $t_{n-q}$ for $q=p, \ldots, n-1$, so we're left with determining $s$. By Theorem 2.3(b), $s$ is uniquely determined by the inequalities

$$
\delta_{n-s}^{(n)} \geq-s \quad \text { and } \quad \delta_{n-s+1}^{(n)} \leq-s-m+n,
$$

and moreover

$$
R^{s \cdot(m-n)} \pi_{*}^{(n)}\left(\mathcal{N}^{(n)}\right)=S_{\tilde{\delta}} F \otimes S_{\delta} G
$$

where $\delta=\delta^{(n)}$ and

$$
\tilde{\delta}=\left(\delta_{1}, \ldots, \delta_{n-s},(-s)^{m-n}, \delta_{n-s+1}+(m-n), \ldots, \delta_{n}+(m-n)\right) .
$$

Since $\delta_{n-t_{1}}^{(n)}=-t_{1}+x_{n} \geq-t_{1}$, it follows as before that $s \leq t_{1}$. Tensoring (3-20) with $\operatorname{det}(F \otimes G)=\operatorname{det}(F)^{\otimes n} \otimes \operatorname{det}(G)^{\otimes m}$ and dualizing, we obtain by putting everything together that there exist integers $0 \leq s \leq t_{1} \leq \cdots \leq t_{n-p} \leq p$ such that

$$
\begin{array}{r}
R^{s \cdot(m-n)+2 \cdot \sum_{j=1}^{n-p} t_{j}} \pi_{*}\left(\bigotimes_{q=p}^{n}\left(\operatorname{det}^{(q)}\right)^{\otimes\left(x_{q}-x_{q+1}\right)} \otimes \operatorname{det}(F \otimes G) \otimes S_{\mu} \mathscr{2}_{p}(F) \otimes S_{\mu} \mathscr{2}_{p}(G)\right)^{*} \\
=S_{\lambda(s)} F \otimes S_{\lambda} G,
\end{array}
$$

where $\lambda(s)$ is defined as in (1-2) and

$$
\lambda_{i}=-m-\delta_{n-i+1} \quad \text { for all } i=1, \ldots, n .
$$


We next check that $\lambda \in W(\underline{x}, p ; \underline{t}, s)$. Since $\mu_{1} \leq-p$, it follows from (3-15) and (3-17) that $\delta_{1} \leq-p+x_{p}$, so $\lambda_{n}=-\delta_{1}-m \geq p-x_{p}-m$; i.e., (3-10a) holds. Letting $i=n$ in (3-18) we get $\delta_{q+1-t_{n-q}}=-t_{n-q}+x_{q+1}$, so $\lambda_{n-q+t_{n-q}}=t_{n-q}-x_{q+1}-m$; i.e., (3-10b) holds. Finally, (3-10c) follows from (3-19).

We conclude from the discussion above that (3-9) holds, after possibly replacing $W(\underline{x}, p ; \underline{t}, s)$ by a smaller set. To see that all weights $\lambda \in W(\underline{x}, p ; \underline{t}, s)$ in fact appear, one has to reverse the steps above in order to show that each $\lambda$ can be reached from a certain weight $\mu$. We give the formula for $\mu$, and leave the details to the interested reader. We first define $\delta \in \mathbb{Z}_{\text {dom }}^{n}$ by reversing (3-21): $\delta_{i}=-m-\lambda_{n+1-i}$. Letting $t_{n-p+1}=p$ and $t_{0}=0$, for each $i=0, \ldots, n-p$ and $j=1, \ldots, t_{i+1}-t_{i}$ we let

$$
\mu_{p-t_{i+1}+j}=\delta_{n-i-t_{i+1}+j}+x_{n-i}+(n-p-i) .
$$

Corollary 3.5. Fix an index $p \in\{0,1, \ldots, n\}$. Suppose that $M$ is an $S$-module with a compatible $\mathrm{GL}(F) \times \mathrm{GL}(G)$ action, admitting a finite filtration with successive quotients isomorphic to $J_{\underline{x}^{j}, p}$ for $j=1, \ldots, r$, where each $\underline{x}^{j}$ is a partition satisfying $x_{1}^{j}=x_{2}^{j}=\cdots=x_{p}^{j}$. We have a decomposition as $\operatorname{GL}(F) \times \mathrm{GL}(G)$-representations

$$
\operatorname{Ext}_{S}^{i}(M, S)=\bigoplus_{j=1}^{r} \operatorname{Ext}_{S}^{i}\left(J_{\underline{x}^{j}, p}, S\right)
$$

for each $i=0,1, \ldots, m \cdot n$. Equivalently, if

$$
0 \longrightarrow A \longrightarrow B \longrightarrow C \longrightarrow 0
$$

is a $\mathrm{GL}(F) \times \mathrm{GL}(G)$-equivariant short exact sequence of $S$-modules admitting filtrations as above, then for each $i=0,1, \ldots, m \cdot n$, the induced sequence

$$
0 \longrightarrow \operatorname{Ext}_{S}^{i}(C, S) \longrightarrow \operatorname{Ext}_{S}^{i}(B, S) \longrightarrow \operatorname{Ext}_{S}^{i}(A, S) \longrightarrow 0
$$

is exact.

Proof. Suppose that the conclusion of the corollary fails, and consider modules $A, B, C$ sitting in an exact sequence (3-23) such that (3-22) holds for $A$ and $C$ but fails for $B$. In particular, not all sequences (3-24) are exact, so there exist an index $i$ and a nontrivial connecting homomorphism

$$
\operatorname{Ext}_{S}^{i}(A, S) \stackrel{\delta}{\longrightarrow} \operatorname{Ext}_{S}^{i+1}(C, S) .
$$

It follows that some irreducible representation of $\mathrm{GL}(F) \times \mathrm{GL}(G)$ appears in both $\operatorname{Ext}_{S}^{i}(A, S)$ and $\operatorname{Ext}_{S}^{i+1}(C, S)$. This is clearly impossible when $m=n$, because from (3-22) and (3-9) it follows that the cohomological degrees $j$ for which $\operatorname{Ext}_{S}^{j}(A, S)$ 
and $\operatorname{Ext}_{S}^{j}(C, S)$ are nonzero satisfy

$$
j \equiv m \cdot n-p^{2}(\bmod 2)
$$

When $m>n$, a similar argument applies: if $\left[S_{\lambda(s)} F \otimes S_{\lambda} G\right]=\left[S_{\mu(t)} F \otimes S_{\mu} G\right]$ (with $\lambda, \mu, \lambda(s)$ and $\mu(t)$ dominant), then it follows from (1-2) that $\lambda=\mu$ and $s=t$; moreover, we get from (3-22) and (3-9) that the cohomological degrees $j$ for which $S_{\lambda(s)} F \otimes S_{\lambda} G$ appears in $\operatorname{Ext}_{S}^{j}(A, S)$ and $\operatorname{Ext}_{S}^{j}(C, S)$ satisfy

$$
j \equiv m \cdot n-p^{2}-s \cdot(m-n)(\bmod 2) .
$$

\section{Ext modules for $S / I_{\underline{x}}$}

In this section we will use the explicit calculation of $\operatorname{Ext}_{S}^{\bullet}\left(J_{x}, p, S\right)$ from the previous section in order to deduce a formula for the characters of $\operatorname{Ext}_{S}^{\bullet}\left(S / I_{\underline{x}}, S\right)$ for all ideals $I_{\underline{x}}$. We begin with an important consequence of the results in the preceding section:

Corollary 4.1. Fix an index $p \in\{0,1, \ldots, n-1\}$ and positive integers $b>c>0$. If we let $\underline{x}=\left(c^{p+1}\right), \underline{y}=\left(b^{p+1}\right)$, then the natural quotient map $S / I_{\underline{y}} \rightarrow S / I_{\underline{x}}$ induces injective maps

$$
\operatorname{Ext}_{S}^{i}\left(S / I_{\underline{x}}, S\right) \hookrightarrow \operatorname{Ext}_{S}^{i}\left(S / I_{\underline{y}}, S\right)
$$

for all $i=0,1, \ldots, m \cdot n$. More generally, if $\underline{z}$ is any partition with $z_{1}=\cdots=z_{p+1}$ and $\underline{x}=\underline{z}+\left(c^{p+1}\right), \underline{y}=\underline{z}+\left(b^{p+1}\right)$, then the quotient map $I_{\underline{z}} / I_{\underline{y}} \rightarrow I_{\underline{z}} / I_{\underline{x}}$ induces injective maps

$$
\operatorname{Ext}_{S}^{i}\left(I_{\underline{z}} / I_{\underline{x}}, S\right) \longleftrightarrow \operatorname{Ext}_{S}^{i}\left(I_{\underline{z}} / I_{\underline{y}}, S\right)
$$

for all $i=0,1, \ldots, m \cdot n$.

Proof. Note that the former statement follows from the latter by taking $\underline{z}=\underline{0}$ and noting that $S=I_{\underline{0}}$. By Lemma 2.2, the modules $A=I_{\underline{x}} / I_{\underline{y}}, B=I_{\underline{z}} / I_{\underline{y}}$, and $C=I_{\underline{z}} / I_{\underline{x}}$ have finite filtrations with quotients isomorphic to $J_{\underline{t}, p}$ for various partitions $\underline{t}$ satisfying $t_{1}=\cdots=t_{p}$. Apply Corollary 3.5 to yield the desired conclusion.

Theorem 4.2. Let $d \geq 0$ and consider partitions $\underline{x}, \underline{y}$, where $\underline{x}$ consists of the first $d$ columns of $y$; i.e., $x_{i}=\min \left(y_{i}, d\right)$ for all $i=1, \ldots, n$. The natural quotient map $S / I_{y} \rightarrow S / I_{\underline{x}}$ induces injective maps

$$
\operatorname{Ext}_{S}^{i}\left(S / I_{\underline{x}}, S\right) \hookrightarrow \operatorname{Ext}_{S}^{i}\left(S / I_{\underline{y}}, S\right)
$$

for all $i=0,1, \ldots, m \cdot n$. 
Proof. Arguing inductively, it suffices to prove the result when all the columns of $y$ outside $\underline{x}$ have the same size (say $p+1$, for $p \in\{0,1, \ldots, n-1\}$ ); i.e., $y=\underline{x}+\left(a^{p+1}\right)$ for some positive integer $a$. Note that this forces $x_{1}=x_{2}=\cdots=x_{p+1}=d$. We prove by descending induction on $p$ that the induced map (4-1) is injective. When $p=n-1$, we have $\underline{x}=\left(d^{n}\right)$ and $y=\left((d+a)^{n}\right)$, so the conclusion follows from Corollary 4.1 (or from the results in [Raicu et al. 2014]).

Suppose now that $p<n-1$ and $\underline{y}=\underline{x}+\left(a^{p+1}\right), x_{1}=\cdots=x_{p+1}=d$. Let $\underline{z}$ be the partition consisting of the columns of $\underline{x}$ of size strictly larger than $p+1$; i.e., $z_{i}=$ $\min \left(x_{i}, x_{p+2}\right)$ for all $i=1, \ldots, n$. Consider the partitions $\underline{\tilde{x}}$ (resp. $\underline{\tilde{y}}$ ), defined by letting $\tilde{x}_{i}=x_{i}\left(\right.$ resp. $\left.\tilde{y}_{i}=y_{i}\right)$ for $i \neq p+2$, and $\tilde{x}_{p+2}=x_{p+1}\left(\right.$ resp. $\left.\tilde{y}_{p+2}=y_{p+1}\right)$. Alternatively, $\underline{\tilde{x}}=\underline{z}+\left(\left(d-x_{p+2}\right)^{p+2}\right), \tilde{y}=\underline{z}+\left(\left(d+a-x_{p+2}\right)^{p+2}\right)$. By induction, for all $i=0,1, \ldots, m \cdot n$, we obtain inclusions

$$
\operatorname{Ext}_{S}^{i}\left(S / I_{\underline{z}}, S\right) \longleftrightarrow \operatorname{Ext}_{S}^{i}\left(S / I_{\underline{\tilde{x}}}, S\right) \text { and } \operatorname{Ext}_{S}^{i}\left(S / I_{\underline{z}}, S\right) \longleftrightarrow \operatorname{Ext}_{S}^{i}\left(S / I_{\underline{\tilde{y}}}, S\right) \text {. }
$$

The natural commutative diagrams

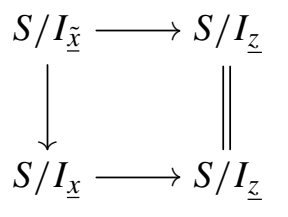

and

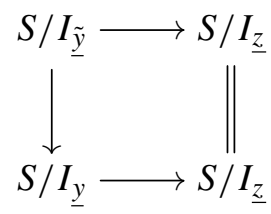

induce commutative diagrams

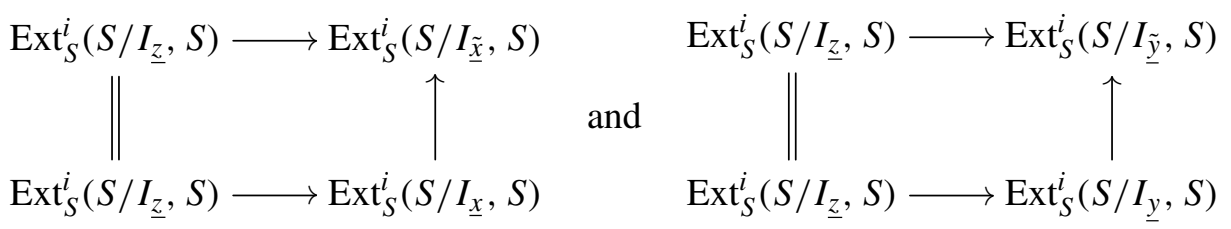

Since the top maps are injective by (4-2), the bottom ones must be injective as well. For all $i=0,1, \ldots, m \cdot n$, we get commutative diagrams

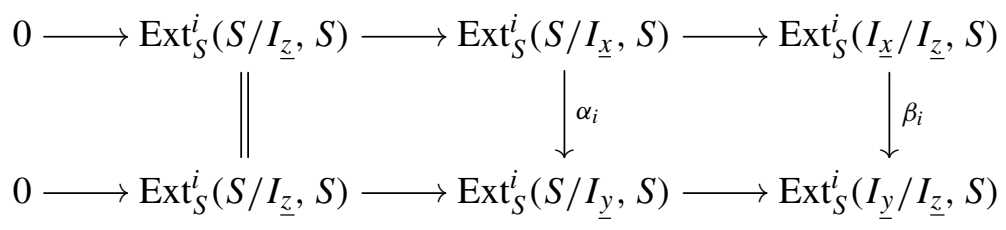

where the rows are exact. The maps $\beta_{i}$ are injective by Corollary 4.1, forcing the maps $\alpha_{i}$ to be injective as well. We conclude that the inclusion (4-1) holds, finishing the proof of the theorem. 
Theorem 4.3. The character of the doubly graded module $\operatorname{Ext}_{S}^{\bullet}\left(S / I_{\underline{x}}, S\right)$ is given by

$$
\begin{aligned}
& \chi_{\operatorname{Ext}_{S}^{\bullet}\left(S / I_{\underline{x}}, S\right)}(z, w) \\
& =\sum_{\substack{1 \leq p \leq n \\
0 \leq s \leq t_{1} \leq \cdots \leq t_{n-p} \leq p-1 \\
\lambda \in W^{\prime}(\underline{x}, p ; \underline{t}, s)}}\left[S_{\lambda(s)} F \otimes S_{\lambda} G\right] \cdot z^{|\lambda|} \cdot w^{m \cdot n+1-p^{2}-s \cdot(m-n)-2 \cdot\left(\sum_{j=1}^{n-p} t_{j}\right)},
\end{aligned}
$$

where $W^{\prime}(\underline{x}, p ; \underline{t}, s)$ is the set of dominant weights $\lambda \in \mathbb{Z}^{n}$ satisfying

$$
\left\{\begin{array}{l}
\lambda_{n} \geq p-x_{p}-m \\
\lambda_{t_{j}+j} \leq t_{j}-x_{n+1-j}-m \quad \text { for } j=1, \ldots, n-p, \\
\lambda_{s} \geq s-n \quad \text { and } \quad \lambda_{s+1} \leq s-m .
\end{array}\right.
$$

Remark 4.4. The condition $t_{n-p} \leq p-1$ in (4-3), combined with the inequalities

$$
t_{n-p}-x_{p+1}-m \geq \lambda_{t_{n-p}+n-p} \geq \lambda_{n} \geq p-x_{p}-m
$$

obtained from (4-4b) by letting $j=n-p$, shows that the only values of $p$ for which there may be a nontrivial contribution to (4-3) are the ones for which $x_{p}>x_{p+1}$ or $p=n$. It follows that for $x_{1}=\cdots=x_{n}$, the only interesting value of $p$ is $p=n$, in which case $I_{\underline{x}}=J_{\underline{x}, n}$ and (4-3) follows from (3-9) and the standard long exact sequence relating $\operatorname{Ext}_{S}^{\bullet}\left(S / I_{\underline{x}}, S\right)$ to $\operatorname{Ext}_{S}^{\bullet}\left(I_{\underline{x}}, S\right)$.

Proof of Theorem 4.3. We induct on the number of columns of $\underline{x}$. When $\underline{x}=\underline{0}$, $S / I_{\underline{x}}=0$, so $\operatorname{Ext}_{S}^{\bullet}\left(S / I_{\underline{x}}, S\right)=0$. It follows that (4-3) is verified in this case, since $W^{\prime}(\underline{0}, p ; \underline{t}, s)$ is empty whenever $0 \leq s \leq p-1$ : to see this, note that

$$
s-m \stackrel{(4-4 \mathrm{c})}{\geq} \lambda_{s+1} \geq \lambda_{n} \stackrel{(4-4 \mathrm{a})}{\geq} p-m
$$

implies $s \geq p$, which is incompatible with the condition $s \leq p-1$.

Suppose now that $y$ is obtained from $\underline{x}$ by appending a column of size $(q+1)$ for some $q=0, \ldots, n-1$. This implies that $x_{1}=\cdots=x_{q+1}$ and $y_{i}=x_{i}+1$ for $1 \leq i \leq q+1$. It follows from Theorem 4.2 that

$$
\chi_{\operatorname{Ext}_{S}^{\bullet}\left(S / I_{\underline{y}}, S\right)}(z, w)=\chi_{\operatorname{Ext}_{S}\left(S / I_{\underline{x}}, S\right)}(z, w)+\chi_{\operatorname{Ext}_{S}^{*}\left(I_{\underline{x}} / I_{\underline{y}}, S\right)}(z, w),
$$

and from Lemma 2.2 and Corollary 3.5 that

$$
\chi_{\operatorname{Ext}_{S}^{*}\left(I_{\underline{x}} / I_{\underline{y}}, S\right)}(z, w)=\sum_{\substack{z=\left(z_{1} \geq \cdots \geq z_{n} \geq 0\right) \\ z_{1}=\cdots=z_{q+1}=x_{1} \\ z_{i} \geq x_{i}, i>q+1}} \chi_{\operatorname{Ext}_{S}^{*}\left(J_{\underline{z}, q}, S\right)}(z, w) .
$$


By Remark 4.4, since $x_{1}=\cdots=x_{q+1}$ and $y_{1}=\cdots=y_{q+1}$, the only relevant terms in (4-3) (for both $x$ and $y$ ) are those for which $p \geq q+1$. For such $p$, $x_{n+1-j}=y_{n+1-j}$ whenever $1 \leq \bar{j} \leq n-p$, so condition (4-4b) is the same for $\underline{x}$ as it is for $\underline{y}$. Equation (4-4c) is clearly the same for both $\underline{x}$ and $\underline{y}$, and the same is true for (4-4a) when $p \geq q+2$. We conclude that $W^{\prime}(\underline{x}, p ; \underline{t}, \bar{s})=W^{\prime}(\underline{y}, p ; \underline{t}, s)$ for $p \neq q+1$, from which it follows using (4-5) and the induction hypothesis that in order to prove (4-3) for $y$, it suffices to show that

$$
\begin{aligned}
& \chi_{\operatorname{Ext}_{\underline{S}}^{*}\left(I_{\underline{x}} / I_{\underline{y}}, S\right)}(z, w) \\
& =\sum_{\substack{p=q+1 \\
0 \leq s \leq t_{1} \leq \cdots \leq t_{n-p} \leq p-1 \\
\lambda \in W^{\prime}(\underline{y}, p ; \underline{t}, s) \backslash W^{\prime}(\underline{x}, p ; \underline{\underline{x}}, s)}}\left[S_{\lambda(s)} F \otimes S_{\lambda} G\right] \cdot z^{|\lambda|} \cdot w^{m \cdot n+1-p^{2}-s \cdot(m-n)-2 \cdot\left(\sum_{j=1}^{n-p} t_{j}\right)} \\
& =\sum_{\substack{0 \leq s \leq t_{1} \leq \cdots \leq t_{n-q-1} \leq q \\
\lambda \in W^{\prime}(\underline{y}, q+1 ; \underline{t}, s) \backslash W^{\prime}(\underline{x}, q+1 ; \underline{t}, s)}}\left[S_{\lambda(s)} F \otimes S_{\lambda} G\right] \cdot z^{|\lambda|} \\
& \cdot w^{m \cdot n-q^{2}-2 \cdot q-s \cdot(m-n)-2 \cdot\left(\sum_{j=1}^{n-q-1} t_{j}\right) .}
\end{aligned}
$$

Note that since (4-4b) and (4-4c) are the same for $\underline{x}$ and $\underline{y}$ when $p=q+1$, it follows that

$\lambda \in W^{\prime}(\underline{y}, q+1 ; \underline{t}, s) \backslash W^{\prime}(\underline{x}, q+1 ; \underline{t}, s)$

$$
\Longleftrightarrow\left\{\begin{array}{l}
\lambda_{n}=q+1-y_{q+1}-m=q-x_{1}-m, \\
\lambda_{t_{j}+j} \leq t_{j}-x_{n+1-j}-m \text { for } j=1, \ldots, n-q-1, \\
\lambda_{s} \geq s-n \text { and } \lambda_{s+1} \leq s-m .
\end{array}\right.
$$

Consider now a partition $\underline{z}$ appearing in (4-6). We claim that $W(\underline{z}, q ; \underline{t}, s)$ is empty unless $t_{n-q}=q$. Furthermore, if $\lambda \in W(\underline{z}, q ; \underline{t}, s)$, then $\lambda_{n}=q-x_{1}-m$. To see this, note that

$$
\lambda_{n} \leq \lambda_{t_{n-q}+n-q} \stackrel{(3-10 \mathrm{~b})}{=} t_{n-q}-z_{q+1}-m=t_{n-q}-z_{q}-m \leq q-z_{q}-m \stackrel{(3-10 \mathrm{a})}{\leq} \lambda_{n},
$$

which forces equalities throughout, and in particular

$$
t_{n-q}=q \quad \text { and } \quad \lambda_{n}=t_{n-q}-z_{q+1}-m=q-x_{1}-m .
$$

We get from (3-9) that

$$
\begin{aligned}
& \chi_{\operatorname{Ext}_{S}^{*}\left(J_{\underline{z}, q}, S\right)}(z, w) \\
& =\sum_{\substack{0 \leq s \leq t_{1} \leq \cdots \leq t_{n-q-1} \leq t_{n-q}=q \\
\lambda \in W(\underline{x}, q ; \underline{\underline{z}}, s)}}\left[S_{\lambda(s)} F \otimes S_{\lambda} G\right] \cdot z^{|\lambda|} \cdot w^{m \cdot n-q^{2}-2 q-s \cdot(m-n)-2 \cdot\left(\sum_{j=1}^{n-q-1} t_{j}\right)} .
\end{aligned}
$$


Combining (4-6), (4-7) and (4-9), it remains to show that

$$
W^{\prime}(\underline{y}, q+1 ; \underline{t}, s) \backslash W^{\prime}(\underline{x}, q+1 ; \underline{t}, s)=\bigcup_{\substack{z=\left(z_{1} \geq \cdots \geq z_{n} \geq 0\right) \\ z_{1}=\cdots=z_{q+1}=x_{1} \\ z_{i} \geq x_{i}, i>q+1}} W(\underline{x}, q ; \underline{t}, s) .
$$

This follows immediately from (4-8) and the fact that the condition

$$
\lambda_{t_{j}+j} \leq t_{j}-x_{n+1-j}-m \text { for } j=1, \ldots, n-q-1
$$

in (4-8) is equivalent to the existence of a partition $\underline{z}$ satisfying $z_{1}=\cdots=z_{q+1}=x_{1}$ and $z_{n+1-j} \geq x_{n+1-j}$ for $j=1, \ldots, n-q-1$ (or equivalently $z_{i} \geq x_{i}$ for $i>q+1$ ), such that

$$
\lambda_{t_{j}+j}=t_{j}-z_{n+1-j}-m \text { for } j=1, \ldots, n-q-1 .
$$

\section{Regularity of the ideals $I_{\underline{x}}$}

The explicit description of the character of $\operatorname{Ext}_{S}^{\bullet}\left(I_{\underline{x}}, S\right)$ obtained in Theorem 4.3 allows us to obtain the following result on the regularity of every ideal $I_{\underline{x}}$, whose proof will be the focus of the current section.

Theorem 5.1. For a partition $\underline{x}$ with at most $n$ parts, letting $x_{n+1}=-1$ we have the following formula for the regularity of the ideal $I_{\underline{x}}$ :

$$
\operatorname{reg}\left(I_{\underline{x}}\right)=\max _{\substack{p=1, \ldots, n \\ x_{p}>x_{p+1}}}\left(n \cdot x_{p}+(p-2) \cdot(n-p)\right) .
$$

In particular, the only ideals $I_{\underline{x}}$ which have a linear resolution are those for which $x_{1}=\cdots=x_{n}$ (i.e., powers $I_{n}^{x_{1}}$ of the ideal $I_{n}$ of maximal minors) or $x_{1}-1=$ $x_{2}=\cdots=x_{n}\left(\right.$ i.e., $\left.I_{n}^{x_{1}-1} \cdot I_{1}\right)$.

By [Eisenbud 1995, Proposition 20.16], one can compute the regularity of a finitely generated $S$-module $M$ by the formula

$$
\operatorname{reg}(M)=\max \left\{-r-j: \operatorname{Ext}_{S}^{j}(M, S)_{r} \neq 0\right\} .
$$

Since $\operatorname{reg}\left(I_{\underline{x}}\right)=\operatorname{reg}\left(S / I_{\underline{x}}\right)+1$, we get by combining (5-2) and (4-3) that

$$
\operatorname{reg}\left(I_{\underline{x}}\right)=\max _{\substack{1 \leq p \leq n \\ 0 \leq s \leq t_{1} \leq \cdots \leq t_{n-p} \leq p-1 \\ \lambda \in W^{\prime}(\underline{x}, p ; \underline{t}, s)}}\left(-|\lambda|-m n+p^{2}+s \cdot(m-n)+2 \cdot\left(\sum_{j=1}^{n-p} t_{j}\right)\right) .
$$

It is then important to decide when $W^{\prime}(\underline{x}, p ; \underline{t}, s)$ is nonempty, which we do in the following lemma: 
Lemma 5.2. Fix $p \in\{1, \ldots, n\}$ and $0 \leq s \leq t_{1} \leq \cdots \leq t_{n-p} \leq p-1$. The set $W^{\prime}(\underline{x}, p ; \underline{t}, s)$ is nonempty if and only if

$$
\left\{\begin{array}{l}
x_{p}-x_{n+1-j} \geq p-t_{j} \quad \text { for } j=1, \ldots,(n-p), \\
s \geq p-x_{p} .
\end{array}\right.
$$

Moreover, the weight $\lambda \in W^{\prime}(\underline{x}, p ; \underline{t}, s)$ of minimal size (i.e., for which the quantity $-|\lambda|$ is maximal) is given by

$$
\left\{\begin{array}{l}
\lambda_{1}=\cdots=\lambda_{s}=(s-n) \\
\lambda_{s+1}=\cdots=\lambda_{n}=\left(p-x_{p}-m\right)
\end{array}\right.
$$

Proof. If $W^{\prime}(\underline{x}, p ; \underline{t}, s)$ is nonempty, then for any $\lambda \in W^{\prime}(\underline{x}, p ; \underline{t}, s)$ we have

$$
t_{j}-x_{n+1-j}-m \stackrel{(4-4 \mathrm{~b})}{\geq} \lambda_{t_{j}+j} \geq \lambda_{n} \stackrel{(4-4 \mathrm{a})}{\geq} p-x_{p}-m
$$

for $j=1, \ldots,(n-p)$ and

$$
s-m \stackrel{(4-4 c)}{\geq} \lambda_{s+1} \geq \lambda_{n} \stackrel{(4-4 a)}{\geq} p-x_{p}-m,
$$

so (5-4) holds.

Conversely, assume that (5-4) holds, and define $\lambda$ via (5-5). It is immediate to check that $\lambda$ satisfies (4-4a)-(4-4c), so $\lambda \in W^{\prime}(\underline{x}, p ; \underline{t}, s)$ and the set is nonempty. The fact that this $\lambda$ has minimal size follows from the fact that any other $\lambda \in$ $W^{\prime}(\underline{x}, p ; \underline{t}, s)$ is dominant and thus satisfies $\lambda_{1} \geq \cdots \geq \lambda_{s} \geq(s-n)$ and $\lambda_{s+1} \geq \cdots \geq$ $\lambda_{n} \geq\left(p-x_{p}-m\right)$, so

$$
|\lambda| \geq s \cdot(s-n)+(n-s) \cdot\left(p-x_{p}-m\right)=(n-s) \cdot\left(p-x_{p}-s-m\right) .
$$

Lemma 5.2 allows us to rewrite (5-3) in the form

$$
\begin{aligned}
\operatorname{reg}\left(I_{\underline{x}}\right)= & \max _{\substack{1 \leq p \leq n \\
0 \leq s \leq t_{1} \leq \cdots \leq t_{n-p} \leq p-1 \\
x_{p}-x_{n+1-j} \geq p-t_{j} \\
s \geq p-x_{p}}}\left(-(n-s) \cdot\left(p-x_{p}-s-m\right)-m n\right. \\
= & \max _{\substack{1 \leq p \leq n \\
0 \leq s \leq t_{1} \leq \cdots \leq t_{n-p} \leq p-1 \\
x_{p}-x_{n+1-j} \geq p-t_{j} \\
s \geq p-x_{p}}}\left(s \cdot\left(p-x_{p}-s\right)+n \cdot\left(x_{p}-p\right)+p^{2}+2 \cdot\left(\sum_{j=1}^{n-p} t_{j}\right)\right) \\
& =\max _{\substack{1 \leq p \leq n \\
0 \leq s \leq p-1 \\
x_{p}-x_{p+1} \geq 1 \\
s \geq p-x_{p}}}\left(s \cdot\left(p-x_{p}-s\right)+n \cdot x_{p}+(p-2) \cdot(n-p)\right) .
\end{aligned}
$$


Since $s \geq p-x_{p}$, we have $s \cdot\left(p-x_{p}-s\right) \leq 0$, with equality if $s=0$ or $s=p-x_{p}$. For $1 \leq p \leq n-1$, the condition $x_{p}-x_{p+1} \geq 1$ forces $x_{p} \geq 1$, so $p-x_{p} \leq p-1$. It follows that we can take $s=\max \left(0, p-x_{p}\right)$ in order to maximize the expression above. Likewise, if $p=n$ and $x_{n} \geq 1$, we take $s=\max \left(0, n-x_{n}\right)$. It follows that when $x_{n} \geq 1$, (5-6) reduces to (5-1). However, if $x_{n}=0$ then for $p=n$ the conditions $s \leq p-1$ and $s \geq p-x_{p}$ are incompatible, so (5-6) reduces to

$$
\operatorname{reg}\left(I_{\underline{x}}\right)=\max _{\substack{p=1, \ldots, n-1 \\ x_{p}>x_{p+1}}}\left(n \cdot x_{p}+(p-2) \cdot(n-p)\right) .
$$

To see that this is the same as (5-1) it suffices to observe that $\operatorname{reg}\left(I_{\underline{x}}\right) \geq n x_{n}=0$ (which is the term corresponding to $p=n$ ).

To finish the proof of the theorem, we need to verify the last assertion. Note that $I_{\underline{x}}$ is generated in degree $x_{1}+\cdots+x_{n}$, so it has a linear resolution if and only if

$$
\operatorname{reg}\left(I_{\underline{x}}\right)=x_{1}+\cdots+x_{n}
$$

When $x_{1}=\cdots=x_{n},(5-1)$ reduces to the term with $p=n$, whose value is $n x_{n}=$ $x_{1}+\cdots+x_{n}$. For $x_{1}-1=x_{2}=\cdots=x_{n}$, the only surviving terms in (5-1) are those with $p=1$ and $p=n$, so we get

$$
\operatorname{reg}\left(I_{\underline{x}}\right)=\max \left(n \cdot\left(x_{1}-1\right)+1, n x_{n}\right)=n \cdot\left(x_{1}-1\right)+1=x_{1}+\cdots+x_{n} .
$$

Conversely, assume that (5-7) holds, and that the $x_{i}$ aren't all equal. Take $p$ minimal with the property that $x_{p}>x_{p+1}$, so that $p<n, x_{1}=\cdots=x_{p}$ and $x_{i} \leq x_{p}-1$ for $i>p$. We have

$$
\begin{aligned}
\operatorname{reg}\left(I_{\underline{x}}\right) & \geq n \cdot\left(x_{p}-p\right)+p^{2}+2 \cdot(p-1) \cdot(n-p) \\
& =p x_{p}+(n-p) \cdot\left(x_{p}-1\right)+(n-p) \cdot(p-1) \geq x_{1}+\cdots+x_{n},
\end{aligned}
$$

with equality when $x_{i}=x_{p}-1$ for $i>p$ and $(n-p) \cdot(p-1)=0$. This forces $p=1$ and $x_{1}-1=x_{2}=\cdots=x_{n}$, concluding the proof of the theorem.

\section{Local cohomology with support in determinantal ideals}

In this section, we prove our main theorem on local cohomology with support in determinantal ideals. Recall that $S=\operatorname{Sym}\left(\mathbb{C}^{m} \otimes \mathbb{C}^{n}\right)$ denotes the polynomial ring of functions on the space of $m \times n$ matrices, $I_{p} \subset S$ is the ideal of $p \times p$ minors of the generic $m \times n$ matrix, and $H_{p}(z, w)$ is the character of the doubly graded module $H_{I_{p}}^{\bullet}(S)$. Recall also the definition (1-3) of $h_{s}(z)$ and the notation (1-4) for Gauss polynomials. 
Theorem 6.1. For each $p=1, \ldots, n$, we have

$$
H_{p}(z, w)=\sum_{s=0}^{p-1} h_{s}(z) \cdot w^{(n-p+1)^{2}+(n-s) \cdot(m-n)} \cdot\left(\begin{array}{c}
n-s-1 \\
p-s-1
\end{array}\right)\left(w^{2}\right) .
$$

To prove the theorem, note that since the system of ideals $\left\{I_{\left(d^{p}\right)}: d \geq 0\right\}$ is cofinal with the one consisting of powers of the ideal of $p \times p$ minors, we obtain from [Eisenbud 2005, Exercise A1D.1] that for each $i=0,1, \ldots, m \cdot n$ we have

$$
H_{I_{p}}^{i}(S)=\underset{d}{\lim } \operatorname{Ext}_{S}^{i}\left(S / I_{\left(d^{p}\right)}, S\right),
$$

where the successive maps in the directed system are induced from the natural quotient maps

$$
S / I_{\left((d+1)^{p}\right)} \rightarrow S / I_{\left(d^{p}\right)} .
$$

By Theorem 4.2 all these maps are injective, so the description of the character of $H_{I_{p}}^{i}(S)$ can be deduced from Theorem 4.3. Note that the partitions $\underline{x}$ to which we apply Theorem 4.3 have the property that $x_{1}=\cdots=x_{p}=d$ and $x_{i}=0$ for $i>p$. Since we are interested in the limit as $d \rightarrow \infty$, we might as well assume that $x_{1}=\cdots=x_{p}=\infty$, in which case (4-4a) becomes vacuous. In what follows, $\lambda$ will always be assumed to be a dominant weight.

If $s \leq t_{j}$ then $s+1 \leq t_{j}+j$ for every $j=1, \ldots, n-p$, so we get

$$
\lambda_{t_{j}+j} \stackrel{\left(\lambda \in \mathbb{Z}_{\text {dom }}^{n}\right)}{\leq} \lambda_{s+1} \stackrel{(4-4 c)}{\leq} s-m \stackrel{\left(s \leq t_{j}\right)}{\leq} t_{j}-m
$$

i.e., (4-4c) implies (4-4b) (note that $x_{n+1-j}=0$ for $j \leq n-p$ ). We conclude that

$$
\begin{gathered}
H_{p}(z, w)=\sum_{\substack{0 \leq s \leq t_{1} \leq \cdots \leq t_{n-p} \leq p-1 \\
\lambda_{s} \leq s-n \\
\lambda_{s+1} \leq s-m}}\left[S_{\lambda(s)} F \otimes S_{\lambda} G\right] \cdot z^{|\lambda|} \cdot w^{m \cdot n+1-p^{2}-s \cdot(m-n)-2 \cdot\left(\sum_{j=1}^{n-p} t_{j}\right)} \\
\stackrel{(1-3)}{=} \sum_{\substack{0 \leq s \leq t_{1} \leq \cdots \leq t_{n-p} \leq p-1}} h_{s}(z) \cdot w^{m \cdot n+1-p^{2}-s \cdot(m-n)-2 \cdot\left(\sum_{j=1}^{n-p} t_{j}\right)},
\end{gathered}
$$

which yields, upon setting $t_{j}^{\prime}:=p-1-t_{j}$,

$$
\begin{aligned}
H_{p}(z, w) & =\sum_{s=0}^{p-1} h_{s}(z) \cdot w^{(n-p+1)^{2}+(n-s) \cdot(m-n)} \cdot \sum_{p-1-s \geq t_{1}^{\prime} \geq \cdots \geq t_{n-p}^{\prime} \geq 0} w^{2 \cdot\left(\sum_{j=1}^{n-p} t_{j}^{\prime}\right)} \\
\stackrel{(1-4)}{=} & \sum_{s=0}^{p-1} h_{s}(z) \cdot w^{(n-p+1)^{2}+(n-s) \cdot(m-n)} \cdot\left(\begin{array}{c}
n-s-1 \\
p-s-1
\end{array}\right)\left(w^{2}\right) .
\end{aligned}
$$




\section{Acknowledgments}

This work was initiated while we were visiting the Mathematical Sciences Research Institute, whose hospitality we are grateful for. Special thanks go to Emily Witt, who participated in the initial stages of this project. We would also like to thank David Eisenbud, Steven Sam, Anurag Singh and Uli Walther for helpful conversations, as well as the anonymous referee for suggesting improvements to the presentation. Experiments with the computer algebra software Macaulay2 [Grayson and Stillman 2013] have provided numerous valuable insights. Raicu acknowledges the support of NSF grant 1303042. Weyman acknowledges the support of the Alexander von Humboldt Foundation and of NSF grant 0901185.

\section{References}

[Akin and Weyman 2007] K. Akin and J. Weyman, "Primary ideals associated to the linear strands of Lascoux's resolution and syzygies of the corresponding irreducible representations of the Lie superalgebra gl $(m \mid n)$ ”, J. Algebra 310:2 (2007), 461-490. MR 2009c:17007 Zbl 1171.17002

[Akin et al. 1981] K. Akin, D. A. Buchsbaum, and J. Weyman, "Resolutions of determinantal ideals: the submaximal minors", Adv. Math. 39:1 (1981), 1-30. MR 82h:13011 Zbl 0474.14035

[Bruns and Schwänzl 1990] W. Bruns and R. Schwänzl, "The number of equations defining a determinantal variety”, Bull. London Math. Soc. 22:5 (1990), 439-445. MR 91k:14035 Zbl 0725.14039

[Bruns and Vetter 1988] W. Bruns and U. Vetter, Determinantal rings, Lecture Notes in Math. 1327, Springer, Berlin, 1988. MR 89i:13001 Zbl 0673.13006

[de Concini et al. 1980] C. de Concini, D. Eisenbud, and C. Procesi, "Young diagrams and determinantal varieties”, Invent. Math. 56:2 (1980), 129-165. MR 81m:14034 Zbl 0435.14015

[Eisenbud 1995] D. Eisenbud, Commutative algebra: with a view toward algebraic geometry, Graduate Texts in Mathematics 150, Springer, New York, 1995. MR 97a:13001 Zbl 0819.13001

[Eisenbud 2005] D. Eisenbud, The geometry of syzygies: a second course in commutative algebra and algebraic geometry, Graduate Texts in Mathematics 229, Springer, New York, 2005. MR 2005h:13021 Zbl 1066.14001

[Fulton and Harris 1991] W. Fulton and J. Harris, Representation theory: a first course, Graduate Texts in Mathematics 129, Springer, New York, 1991. MR 93a:20069 Zbl 0744.22001

[Grayson and Stillman 2013] D. R. Grayson and M. E. Stillman, "Macaulay 2: a software system for research in algebraic geometry", 2013, Available at http://www.math.uiuc.edu/Macaulay2.

[Hartshorne 1977] R. Hartshorne, Algebraic geometry, Graduate Texts in Mathematics 52, Springer, New York, 1977. MR 57 \#3116 Zbl 0367.14001

[Hochster and Eagon 1971] M. Hochster and J. A. Eagon, "Cohen-Macaulay rings, invariant theory, and the generic perfection of determinantal loci”, Amer. J. Math. 93 (1971), 1020-1058. MR 46 \#1787 Zbl 0244.13012

[Lyubeznik et al. 2013] G. Lyubeznik, A. Singh, and U. Walther, "Local cohomology modules supported at determinantal ideals", preprint, 2013. arXiv 1308.4182

[Peskine and Szpiro 1973] C. Peskine and L. Szpiro, "Dimension projective finie et cohomologie locale: applications à la démonstration de conjectures de M. Auslander, H. Bass et A. Grothendieck", Inst. Hautes Études Sci. Publ. Math. 42 (1973), 47-119. MR 51 \#10330 Zbl 0268.13008 
[Raicu et al. 2014] C. Raicu, J. Weyman, and E. E. Witt, "Local cohomology with support in ideals of maximal minors and sub-maximal Pfaffians", Adv. Math. 250 (2014), 596-610. MR 3122178 Zbl 06284419

[Weyman 2003] J. Weyman, Cohomology of vector bundles and syzygies, Cambridge Tracts in Mathematics 149, Cambridge University Press, 2003. MR 2004d:13020 Zbl 1075.13007

[Witt 2012] E. E. Witt, "Local cohomology with support in ideals of maximal minors", Adv. Math. 231:3-4 (2012), 1998-2012. MR 2964631 Zbl 1253.13019

Communicated by David Eisenbud

Received 2013-09-27 Revised 2014-02-25 Accepted 2014-03-26

craicu@nd.edu

Department of Mathematics, University of Notre Dame, 255 Hurley Hall, Notre Dame, IN 46556, United States

Simion Stoilow Institute of Mathematics of the Romanian Academy, 21 Calea Grivitei Street, 010702 Bucharest, Romania

jerzy.weyman@uconn.edu Department of Mathematics, University of Connecticut, Storrs, CT 06269, United States 


\section{Algebra \& Number Theory}

msp.org/ant

\section{EDITORS}

MANAGING EDITOR

Bjorn Poonen

Massachusetts Institute of Technology

Cambridge, USA

\author{
EDITORIAL BOARD CHAIR \\ David Eisenbud \\ University of California \\ Berkeley, USA
}

BOARD OF EDITORS

Georgia Benkart

Dave Benson

Richard E. Borcherds

John H. Coates

J-L. Colliot-Thélène

Brian D. Conrad

Hélène Esnault

Hubert Flenner

Edward Frenkel

Andrew Granville

Joseph Gubeladze

Roger Heath-Brown

Craig Huneke

Yujiro Kawamata

János Kollár

Yuri Manin

Barry Mazur

Philippe Michel

Susan Montgomery
University of Wisconsin, Madison, USA

University of Aberdeen, Scotland

University of California, Berkeley, USA

University of Cambridge, UK

CNRS, Université Paris-Sud, France

University of Michigan, USA

Freie Universität Berlin, Germany

Ruhr-Universität, Germany

University of California, Berkeley, USA

Université de Montréal, Canada

San Francisco State University, USA

Oxford University, UK

University of Virginia, USA

University of Tokyo, Japan

Princeton University, USA

Northwestern University, USA

Harvard University, USA

École Polytechnique Fédérale de Lausanne

University of Southern California, USA
Shigefumi Mori

Raman Parimala

Jonathan Pila

Anand Pillay

Victor Reiner

Peter Sarnak

Joseph H. Silverman

Michael Singer

Vasudevan Srinivas

J. Toby Stafford

Bernd Sturmfels

Richard Taylor

Ravi Vakil

Michel van den Bergh

Marie-France Vignéras

Kei-Ichi Watanabe

Efim Zelmanov

Shou-Wu Zhang
RIMS, Kyoto University, Japan

Emory University, USA

University of Oxford, UK

University of Notre Dame, USA

University of Minnesota, USA

Princeton University, USA

Brown University, USA

North Carolina State University, USA

Tata Inst. of Fund. Research, India

University of Michigan, USA

University of California, Berkeley, USA

Harvard University, USA

Stanford University, USA

Hasselt University, Belgium

Université Paris VII, France

Nihon University, Japan

University of California, San Diego, USA

Princeton University, USA

\section{PRODUCTION}

production@msp.org

Silvio Levy, Scientific Editor

See inside back cover or msp.org/ant for submission instructions.

The subscription price for 2014 is US $\$ 225 /$ year for the electronic version, and $\$ 400 /$ year ( $\$ 55$, if shipping outside the US) for print and electronic. Subscriptions, requests for back issues and changes of subscribers address should be sent to MSP.

Algebra \& Number Theory (ISSN 1944-7833 electronic, 1937-0652 printed) at Mathematical Sciences Publishers, 798 Evans Hall \#3840, c/o University of California, Berkeley, CA 94720-3840 is published continuously online. Periodical rate postage paid at Berkeley, CA 94704, and additional mailing offices.

ANT peer review and production are managed by EditFLOw ${ }^{\circledR}$ from Mathematical Sciences Publishers.

\section{PUBLISHED BY}

mathematical sciences publishers

nonprofit scientific publishing

http://msp.org/

(C) 2014 Mathematical Sciences Publishers 


\section{Algebra \& Number Theory}

Volume $8 \quad$ No. $5 \quad 2014$

Polarization estimates for abelian varieties

DAVID MASSER and GISBERT WÜSTHOLZ

Compatibility between Satake and Bernstein isomorphisms in characteristic $p$

1071

RACHEL OLLIVIER

The final $\log$ canonical model of $\bar{M}_{6}$

1113

FABIAN MÜLLER

Poisson structures and star products on quasimodular forms

1127

FRANÇOIS DUMAS and EMMANUEL ROYER

Affinity of Cherednik algebras on projective space

GWYN BELLAMY and MAURIZIO MARTINO

ALEXANDRU CHIRVASITU

Tetrahedral elliptic curves and the local-global principle for isogenies

1201

BARINDER S. BANWAIT and JOHN E. CREMONA

Local cohomology with support in generic determinantal ideals

1231

Claudiu RAICU and JERZY WEYMAN

Affine congruences and rational points on a certain cubic surface

1259

PIERRE LE BoudeC 